\title{
MyD88-dependent dendritic and epithelial cell crosstalk orchestrates immune responses to allergens
}

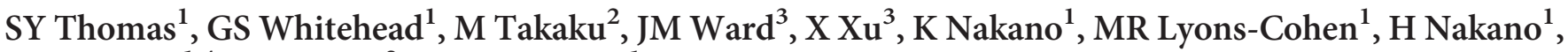 \\ KM Gowdy ${ }^{1,4}$, PA Wade ${ }^{2}$ and DN Cook ${ }^{1}$
}

\begin{abstract}
Sensitization to inhaled allergens is dependent on activation of conventional dendritic cells (cDCs) and on the adaptor molecule, MyD88. However, many cell types in the lung express Myd88, and it is unclear how signaling in these different cell types reprograms cDCs and leads to allergic inflammation of the airway. By combining ATAC-seq with RNA profiling, we found that MyD88 signaling in CDCs maintained open chromatin at select loci even at steady state, allowing genes to be rapidly induced during allergic sensitization. A distinct set of genes related to metabolism was indirectly controlled in cDCs through MyD88 signaling in airway epithelial cells (ECs). In mouse models of asthma, Myd88 expression in ECs was critical for eosinophilic inflammation, whereas Myd88 expression in cDCs was required for Th17 cell differentiation and consequent airway neutrophilia. Thus, both cell-intrinsic and cell-extrinsic MyD88 signaling controls gene expression in cDCs and orchestrates immune responses to inhaled allergens.
\end{abstract}

Asthma is a chronic disease of the airways characterized by reversible airway obstruction, airway hyperresponsiveness and inflammation. This disease is now regarded as a heterogeneous set of lung pathologies that vary with regard to the provoking stimulus, age of onset, type of inflammation, mucus production, lung function, and response to treatment. ${ }^{1}$ Whereas many patients display classic, type 2 cytokine-associated eosinophilic inflammation and are generally responsive to steroid-responsive inhaled corticosteroids, approximately half of patients with asthma have non-eosinophilic forms of the disease, often with neutrophilic inflammation of the airway. ${ }^{2-4}$ These patients are typically resistant to inhaled corticosteroids and can develop uncontrolled, life-threatening disease. ${ }^{5}$ Several lines of evidence suggest that interleukin (IL)-17-producing $\mathrm{T}$ helper type 17 (Th17) cells, which are steroid-resistant, ${ }^{6,7}$ might drive the latter form of asthma by promoting recruitment of neutrophils to the airway. ${ }^{7-13}$

The initiation of adaptive immunity, including Th2 and Th17 responses to inhaled allergens, is dependent on Cd11c- expressing, conventional dendritic cells (cDCs). ${ }^{14}$ Multiple receptors on the surface of $\mathrm{cDCs}$ allow them to directly sense a variety of allergens and their associated microbial products, and to respond by increasing cytokine production and display of cell surface co-stimulatory molecules. This direct sensing of pathogen-associated microbial patterns is undoubtedly important for their function, but some features of cDCs might also be indirectly controlled by signals provided by other cells. To test this, bone marrow chimeric mice are frequently generated to compare gene function in radio-resistant and -sensitive cells, and this approach was used to show Toll-like receptor 4 (TLR4) signaling in airway structural cells is required for allergic sensitization to lipopolysaccharide-containing preparations of house dust mite (HDM) allergen. ${ }^{15}$ Lung epithelial cells (ECs) are often presumed to be the major radio-resistant cell type that modifies the function of lung cDCs, but lung macrophages are also largely resistant to irradiation, which confounds interpretation of this type of experiment. ${ }^{16}$ Furthermore, radiation induces profound transcriptional

${ }^{1}$ Immunity, Inflammation and Disease Laboratory, National Institute of Environmental Health Sciences, NIH, Research Triangle Park, North Carolina, USA. ${ }^{2}$ Embryonic Stem Cell and Chromatin Biology Laboratory, National Institute of Environmental Health Sciences, NIH, Research Triangle Park, North Carolina, USA. ${ }^{3}$ Integrated Bioinformatics, National Institute of Environmental Health Sciences, NIH, Research Triangle Park, North Carolina, USA and ${ }^{4}$ Department of Pharmacology \& Toxicology, Brody School of Medicine at East Carolina University, Greenville, North Carolina, USA. Correspondence: SY Thomas (thomassy@niehs.nih.gov) 
changes in ECs that have unknown effects on their function. ${ }^{17}$ Thus, a major gap exists in our understanding of how direct and indirect recognition of pathogen-associated microbial patterns contribute to the epigenetic landscape, transcriptional profiles, and activities of cDCs in vivo.

Myeloid differentiation primary response gene 88 (MyD88) is an adaptor molecule used by all known TLRs, except TLR3. MyD88 is required for allergic sensitization through the airway in several models of asthma, including when lipopolysaccharide or flagellin (FLA) is used as an adjuvant to promote allergic responses to ovalbumin (OVA) ${ }^{18,19}$ To better understand cDC activation during allergic sensitization without the confounding aspects of irradiation, we used a genetic approach to selectively delete $M y d 88$ in either ECs or Cd11c-expressing cells, and sensitized the animals through the airway with OVA/ FLA, which is strictly dependent on MyD88. ${ }^{19}$ Myd88 expression in each of these cell types contributed to chromatin accessibility and gene expression in cDCs. Phenotypically, Myd88 expression in ECs was required for robust eosinophilic inflammation, whereas expression of this gene in Cd11cexpressing cells was required for Th17-associated neutrophilic inflammation. These findings reveal that reprogramming of lung $\mathrm{cDCs}$ and consequent induction of allergic pulmonary inflammation results from both cell-intrinsic and cell-extrinsic MyD88-dependent signaling.

\section{RESULTS}

\section{Generation of mice lacking Myd88 in Cd11c-expressing cells}

To understand how MyD88 signaling in cDCs affects their gene expression and thus allergic sensitization through the airway, we employed transgenic mice expressing Cre from the $C d 11 c$ promoter. ${ }^{20} \mathrm{We}$ first confirmed cell specificity by crossing these animals to tdTomato reporter mice in which a LoxP-flanked transcriptional stop cassette (LoxP-Stop-LoxP) is positioned upstream of a tandem dimer $(t d)$ Tomato gene at the constitutively active Rosa26 locus. Precision cut lung slices from offspring of this cross confirmed the presence of tdTomato-expressing cells around the airways and within the lung parenchyma. Staining of the precision cut lung slices with antibodies against CD103 showed that one of the two major types of cDCs in the lung, $\mathrm{CD}_{103}{ }^{+} \mathrm{cDCs}$, are closely associated with the abluminal surface of the airway wall (Figure 1a, top panels). Precision cut lung slice staining with antibodies to SIRP-1 $\alpha$, which identify CD11b $\mathrm{bDCs}^{\mathrm{hi}}$ and macrophages, showed that these cells reside throughout the lung parenchyma (Figure 1a, bottom panels). Flow cytometric analysis showed that $>90 \%$ of CD $103^{+} \mathrm{cDCs}, \mathrm{CD} 11 \mathrm{~b}^{+} \mathrm{cDCs}$, and alveolar macrophages (AMs) expressed tdTomato (Supplementary Figure 1a,b online). Having confirmed the specificity of Cd11c-cre expression, we crossed Cd11c-cre mice to $M y d 88^{f x / f x}$ mice, which bear a LoxP-flanked version of the third exon of $M y d 88 .^{22}$ Using quantitative PCR, we confirmed that purified cDCs and AMs from $M y d 88^{f x / f x} \mathrm{CD} 11 \mathrm{c}$-cre mice (hereafter called DC-KO mice), had undergone deletion of the third exon, whereas it was retained in CDCs and AMs of

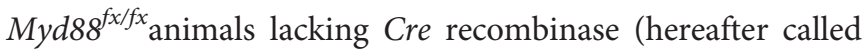
WT mice) (Figure 1b).

\section{Generation of mice lacking Myd88 in ECs}

To study how MyD88 signaling in ECs affects immune responses, including gene expression and function of lung cDCs, we used transgenic mice expressing Cre under control of the human surfactant protein C (Sftpc) promoter. ${ }^{23}$ Although Sftpc is not expressed in most mature ECs, it is expressed in their precursors, and genetic loci that undergo Cre-mediated recombination at that stage are reported to remain recombined in mature ECs. ${ }^{24}$ To confirm EC-specific, Cre-mediated recombination, we bred Sftpc-cre mice to the Cre-inducible tdTomato reporter strain. Tiled microscopic analysis of lung sections revealed fluorescent ECs throughout the entire respiratory tree of these animals, including the trachea, bronchi, bronchioles, and alveoli (Figure 1c). By comparing adjacent serial sections by fluorescent microscopy and hematoxylin and eosin staining, we confirmed that the tdTomato fluorescence was restricted to ECs and did not include endothelial cells lining the blood vessels (data not shown). Flow cytometric analysis confirmed that $>90 \%$ of ECs expressed tdTomato (Supplementary Figure 1c,d), whereas less than $0.1 \%$ of Lin $^{+}$Epithelial cell adhesion molecule (EpCAM) $)^{-}$cells did. Having established that the Sftpc-cre transgene efficiently targets ECs, we bred Sftpc-cre mice to $M y d 88^{f x / f x}$ mice. Sorted

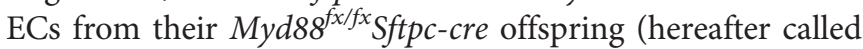
EC-KO mice) had very little Myd88 mRNA compared with ECs from WT or DC-KO mice (Figure 1d).

\section{Myd88 expression in Cd11c-expressing cells and ECs controls neutrophilia and eosinophilia, respectively, in mouse models of asthma}

We next studied the role of cell-specific MyD88 in two different animal models of asthma. In the first model, we sensitized mice by allowing them to inhale OVA/FLA, then challenged the animals by exposing them to aerosolized OVA (Figure 2a). Following OVA challenge, DC-KO mice had markedly reduced neutrophilic inflammation compared to WT mice, whereas eosinophilic inflammation was similar in these two strains. Contrasting results were seen with EC-KO mice, which had markedly reduced eosinophilic inflammation compared to WT mice but only a modest reduction in neutrophils. Almost identical results were obtained in the second model of asthma in which an extract of HDMs was used to sensitize and challenge the animals (Figure $\mathbf{2 b}$ ). Together, these results show that Myd88 expression in Cd11c-expressing cells is important for neutrophilic inflammation in these models of asthma, whereas Myd88 expression in ECs is critical for eosinophilic inflammation. While MyD88 is required for signaling responses to FLA, it is also required for responses to IL-33, which has been associated with some types of allergic responses. ${ }^{25}$ As expected, Myd88 null mice failed to become sensitized to OVA when IL-33 was used as an adjuvant, and they did not develop inflammation upon subsequent OVA challenge (Supplementary Figure 2a). By contrast, WT, DC$\mathrm{KO}$ and EC-KO mice all displayed robust neutrophilic and 
a
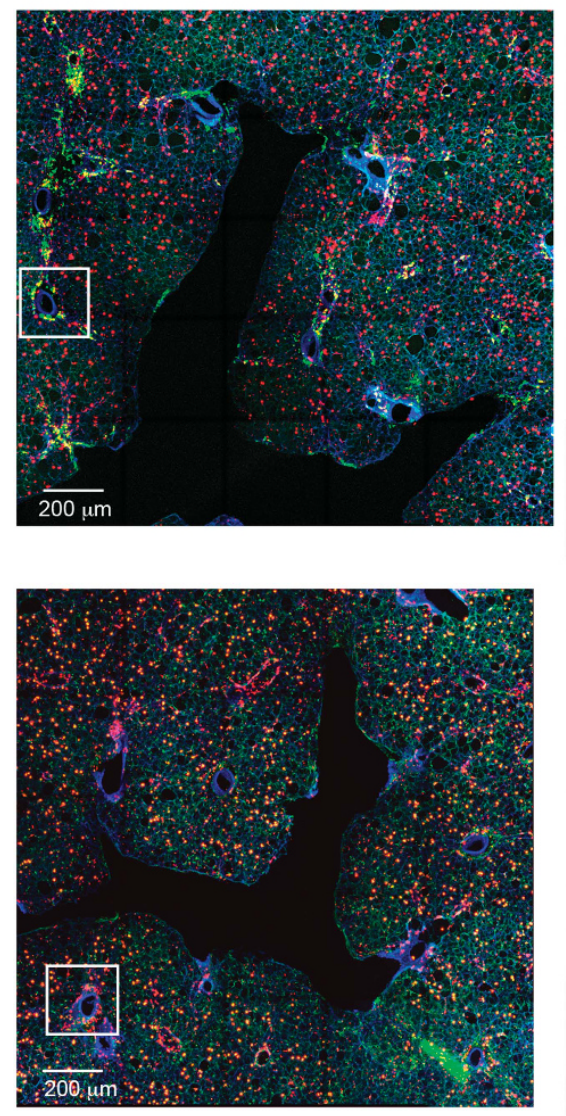

b

Myd88 exon 3 in cDCs

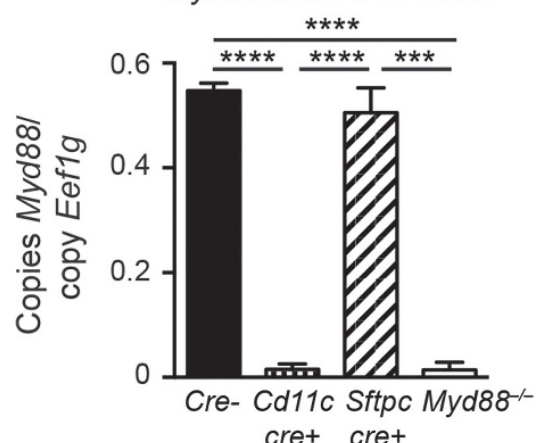

c

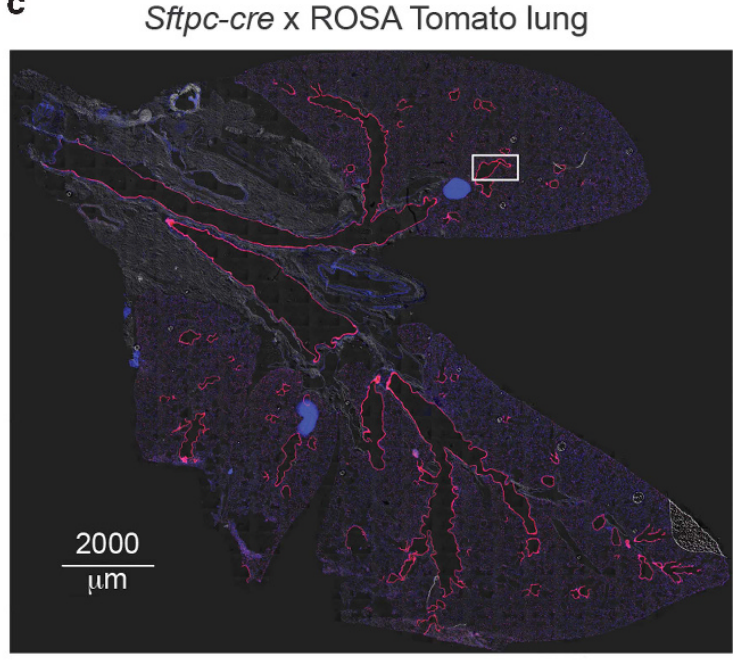

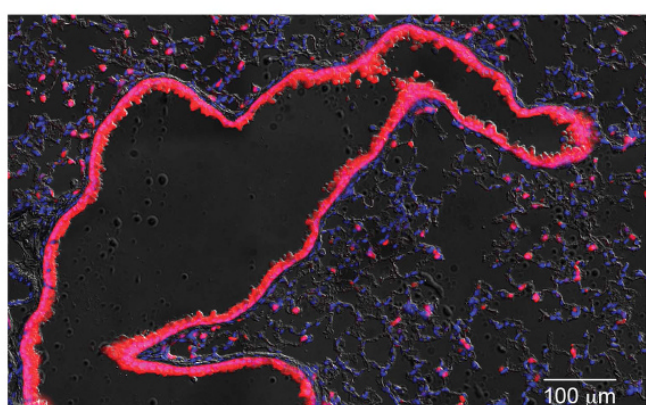

SFTPC Cre x ROSA Tomato

DAPI

DIC/ brightfield

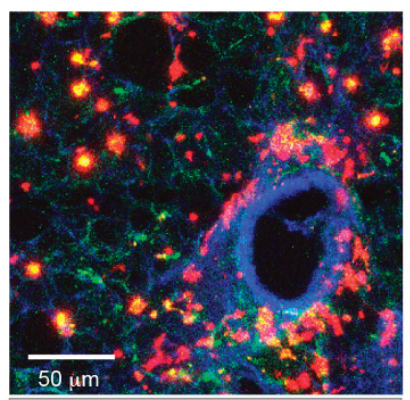

CD11c cre x ROSA Tomato SIRP1 $\alpha^{*}$

E-cadherin*

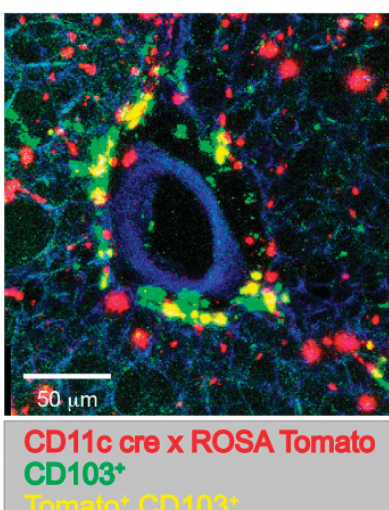

E-cadherin ${ }^{+}$

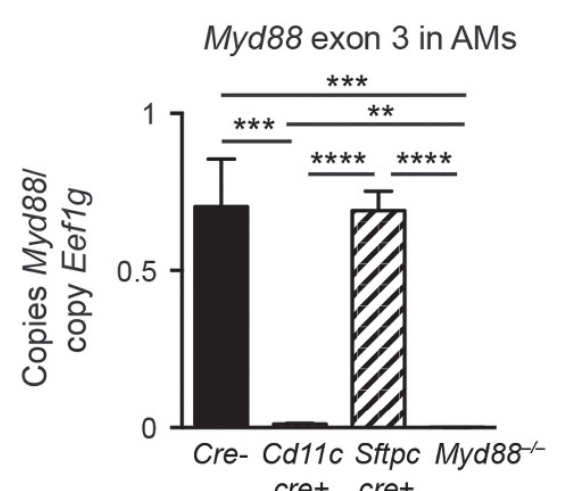

d

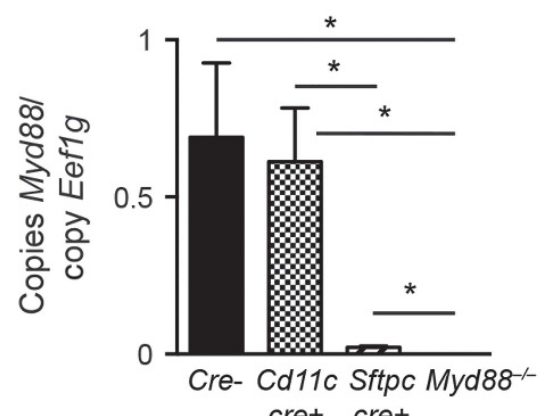

Figure 1 Cd11c drives Cre-mediated recombination in lung DCs and AMs while Sftpc drives Cre-mediated recombination in ECs. (a) Fluorescent microscopic images of a PCLS prepared from progeny of Cd11c-cre x LoxP-Stop-LoxP-tdTomato cross. Top images show Tomato ${ }^{+}$cells (red), CD103 ${ }^{+}$ (green), Tomato ${ }^{+} \mathrm{CD}_{103}{ }^{+} \mathrm{DCs}$ (yellow), and E-cadherin ${ }^{+}$ECs (blue). Bottom images show Tomato ${ }^{+}$cells (red), SIRP- $1 \alpha^{+}$(green), Tomato ${ }^{+}$SIRP$1 \alpha^{+}$DCs (yellow), and E-cadherin ${ }^{+}$ECs (blue). Low power images (left) include white squares marking an area also shown in higher power (right).

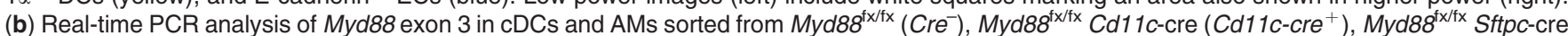

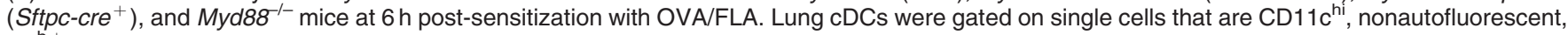
$\mathrm{I}-\mathrm{A}^{\mathrm{b}+}, \mathrm{Ly}_{6 \mathrm{C}^{-}}$, and CD88- Data are representative of 3-6 separate CDC or AM sorts per genotype and are normalized to the housekeeping gene. (c) Fluorescent microscopic images of a frozen lung section from progeny of Sftpc-crex LoxP-Stop-LoxP-tdTomato cross. Shown are a low power image of the whole lung, including the bifurcation of the trachea (top), and a high power image of a representative small airway, showing fluorescent ECs in the airway and alveoli (bottom). tdTomato cells (red), DAPI (blue), and Differential Interference Contrast (DIC)/ brightfield (grey). (d) Real-time PCR for the

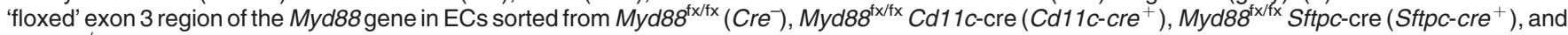
$M y d 88^{-1-}$ mice at $2 \mathrm{~h}$ post-sensitization with OVA/FLA. Data are representative of three different EC sorts per genotype. ${ }^{*} P<0.05,{ }^{* \star} P<0.01,{ }^{*}$ ${ }^{\star \star \star} P<0.001,{ }^{* \star \star \star} P<0.0001$. AM, alveolar macrophage; $\mathrm{CDC}$, conventional dendritic cells; EC, epithelial cells; FLA, flagellin; OVA, ovalbumin; PCLS, precision cut lung slices. 
a
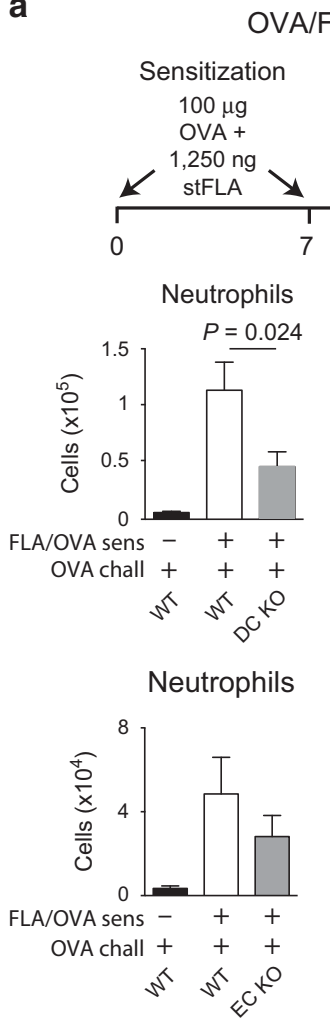

OVA/FLA model

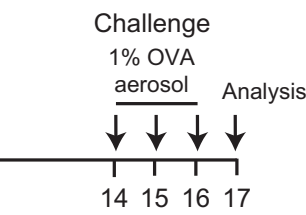

Eosinophils

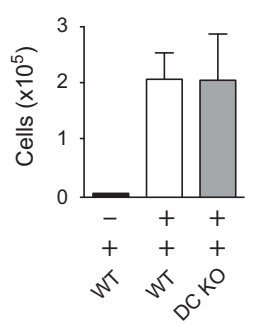

Eosinophils

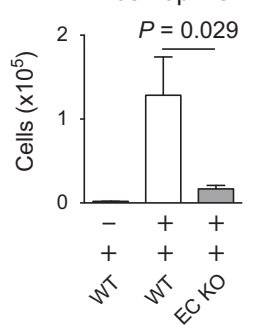

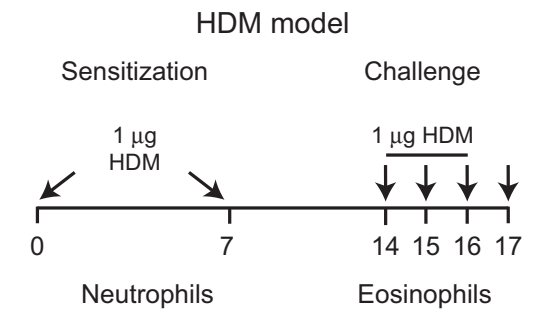
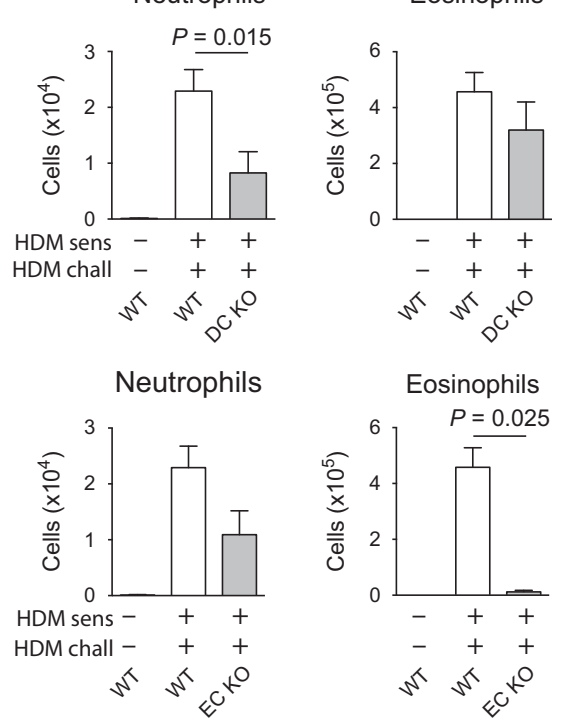

Figure $2 \mathrm{DC}$ and EC expression of MyD88 control distinct facets of allergic pulmonary inflammation. (a,b) Schematic of OVA/FLA (a) and HDM (b) mouse models of allergic asthma (top). Neutrophilic and eosinophilic inflammation of the airway in WT and DC-KO mice (middle) or WT and EC-KO mice (bottom) sensitized with OVA/FLA and challenged with OVA (a), or sensitized and challenged with HDM (b). Data shown are representative of two experiments, $n=9-12$ mice per group (a,b). DC, dendritic cell; EC, epithelial cell; FLA, flagellin; HDM, house dust mite; OVA, ovalbumin; WT, wild type.

eosinophilic inflammation. This suggests that in the OVA/FLA and HDM models of asthma, MyD88 is required in ECs and Cd11c-expressing cells for responsiveness to TLR ligands, not IL-33.

\section{Myd88 expression in ECs drives early immune responses during allergic sensitization}

We next sought to obtain a molecular understanding of how Myd88 expression in Cd11c-expressing cells and ECs controls the expression of immune response genes during allergic sensitization through the airway. Following instillation of OVA/FLA, the expression of 547 known immune response genes was evaluated in whole lungs of the various recombinant mouse strains using the Nanostring Mouse Immunology Codeset (Supplementary Resource). Between group analyses of the data revealed that at steady state, gene expression at the whole lung level was similar in WT, EC$\mathrm{KO}, \mathrm{DC}-\mathrm{KO}$ and Myd88 null mice (Figure 3a), with only a small number of genes being affected by the absence of MyD88 (Figure 3b, top, and Supplementary Table 1). However, by $2 \mathrm{~h}$ after allergic sensitization with OVA/FLA, genotype-specific differences in gene expression became apparent, with the greatest difference seen between WT and Myd88 null mice (Figure 3a and Supplementary Table 2, left). The expression pattern observed for EC-KO mice was intermediate between the patterns of WT mice and Myd88 null mice, whereas expression in DC-KO mice was similar to that of WT mice (Figure 3b, bottom, and Supplementary Table 3). ECs were also purified from the lung using flow cytometry-based sorting (Figure 3c) and analyzed. In agreement with data from the whole lung, ECs from EC-KO and Myd88 null mice displayed similar expression profiles, but were distinct from those of ECs from WT and DC-KO mice (Figures 3d and e and Supplementary Table 4). Ccl20, Csf2, and Il4ra were among the genes differentially expressed (above the minimum threshold intensity, $\geqslant 1.5$ fold difference between groups, $P<0.01$; see Supplementary Methods) between EC-KO and WT mice at the whole lung level (Figure 3f) and in sorted ECs (Figure 3g). Although the epithelial-associated cytokines, Il25, Il33, and Tslp, have been linked to allergic pulmonary inflammation in some studies, ${ }^{25}$ none of these genes were differentially expressed among the various strains (Figures $3 \mathbf{f}$ and $\mathbf{g}$ ). Loss of MyD88 in Cd11c-expressing cells did not affect gene expression in ECs. Together, these data suggest that shortly after inhalation of OVA/FLA, expression of select proinflammatory genes in ECs is increased in a manner dependent on MyD88 signaling in those cells.

\section{Myd88 expression in both DCs and ECs controls gene transcription in lung DCs at $6 \mathrm{~h}$ post-sensitization}

To determine if MyD88 signaling in Cd11c-expressing cells was important for gene expression in the lung at later time 


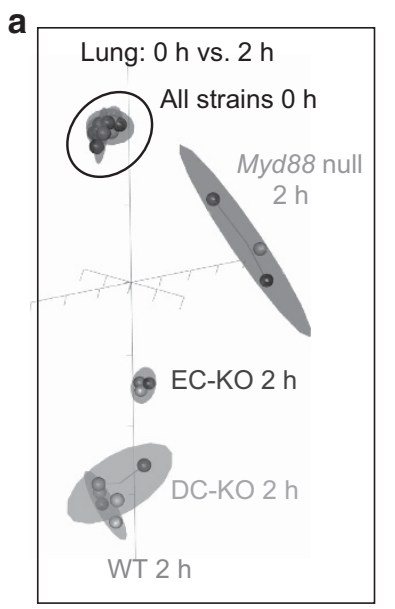

b
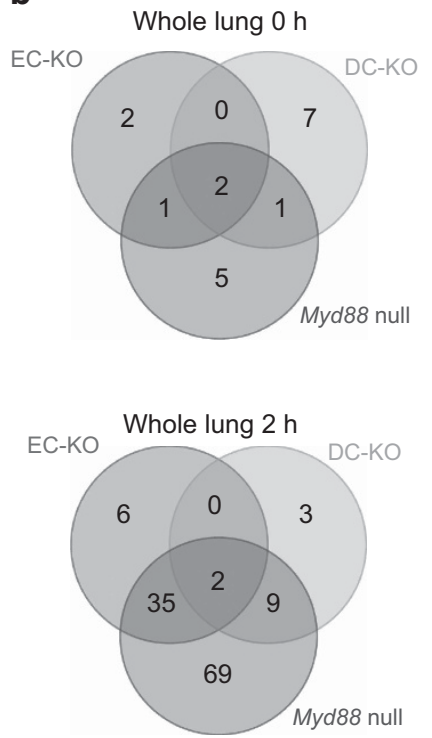

C
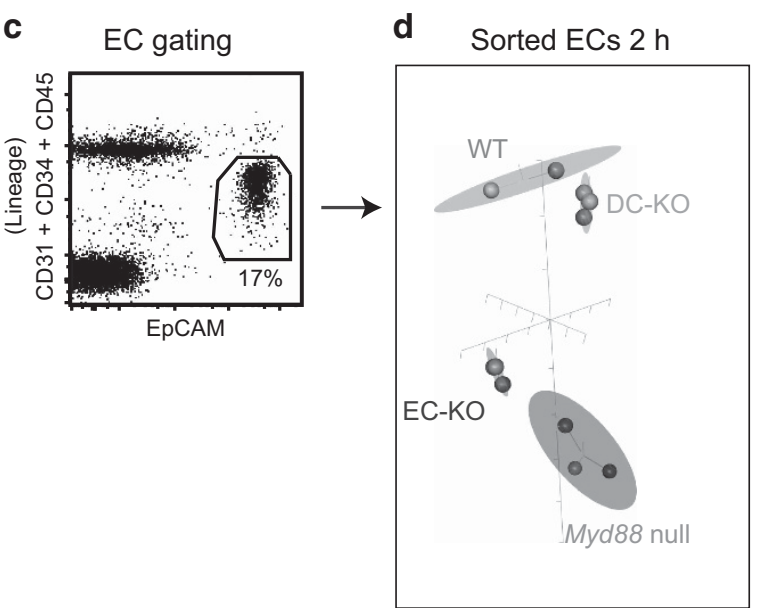

e

ECs $2 \mathrm{~h}$

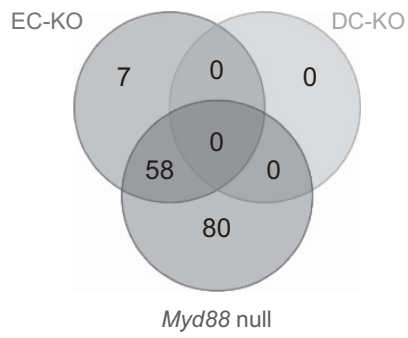

f
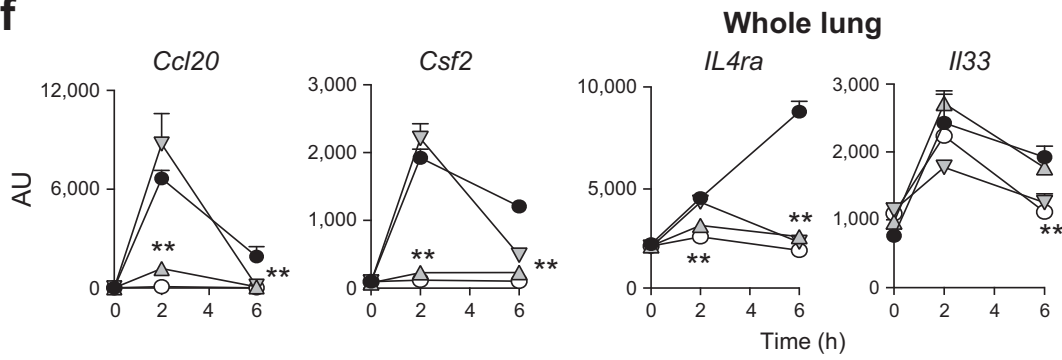

- WT

$\triangle$ ECKO

$\nabla$ DC KO

○. Myd88 null

g

Sorted ECs 2 h
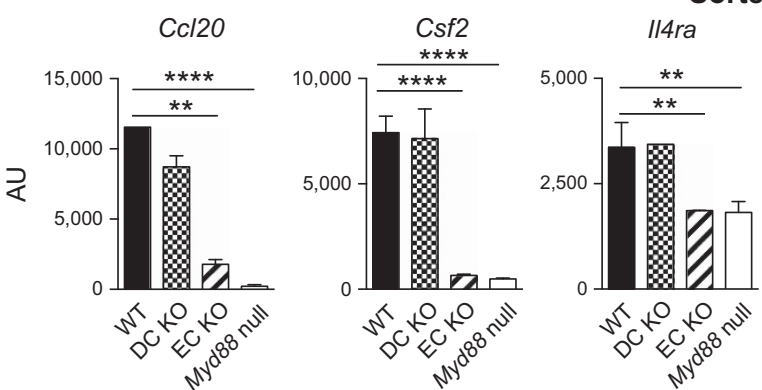

II33

$T s / p$

1125

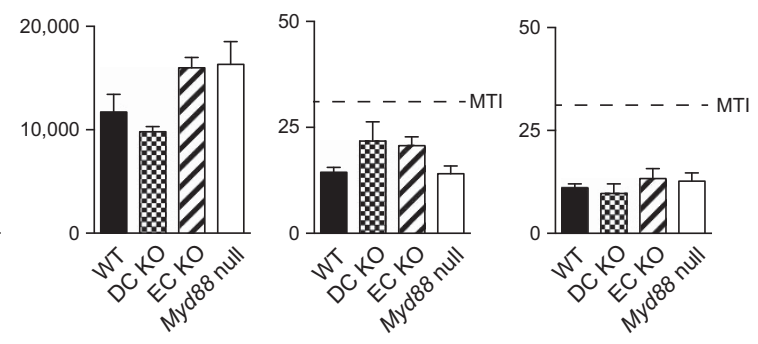

Figure 3 Immune gene expression in whole lung and $\mathrm{ECs}$ at $2 \mathrm{~h}$ post-sensitization. (a) BGA plot of immune gene expression in whole lung at 0 and $2 \mathrm{~h}$ after OVA/FLA-mediated sensitization of the indicated mouse strains. (b) Venn diagram showing the number of genes differentially expressed ( $\geqslant 1.5$ fold; $P \leqslant 0.01, \mathrm{MTI} \geqslant 32$ for at least one group) between lungs of WT mice (reference group) and the indicated mouse strains at 0 (top) and $2 \mathrm{~h}$ (bottom) postsensitization. (c) Gating strategy for purification of ECs. (d) BGA plot of immune gene expression in sorted ECs of the indicated mouse strains at $2 \mathrm{~h}$ postsensitization. (e) Venn diagrams showing the number of genes differentially expressed between sorted ECs of WT mice and the indicated strains ( $\geqslant 1.5$ fold; $P \leqslant 0.01, \mathrm{MTI} \geqslant 32$ for at least one group). $(\mathbf{f}-\mathbf{g})$ Expression of genes in whole lung (f) and ECs at $2 \mathrm{~h}$ post-sensitization $(\mathbf{g})$. $n=3$ mice per group. ${ }^{* *} P<0.01,{ }^{* * *} P<0.0001$. BGA, Between group analyses; EC, epithelial cells; MTI, minimum threshold intensity for gene detection; WT, wild type. A full color version of this figure is available at the Mucosal Immunology journal online.

points during sensitization, we studied RNA prepared from WT, DC-KO, EC-KO, and Myd88 null mice at $6 \mathrm{~h}$ postsensitization (Supplementary Table 2, right). Many of the genes expressed at $6 \mathrm{~h}$ were primarily dependent on MyD88 signaling in Cd11c-expressing cells (Figures $4 \mathbf{4 a}$ and $\mathbf{b}$ and Supplementary Table 5). These genes included Tnf 


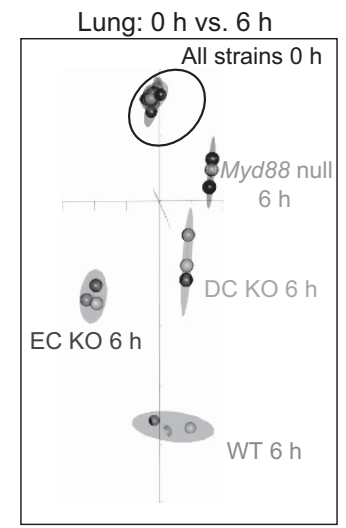

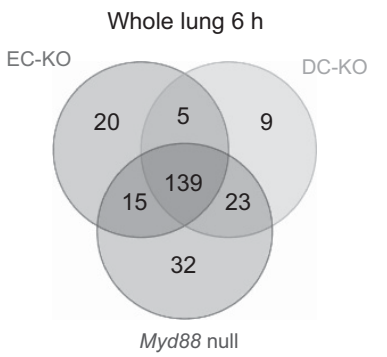

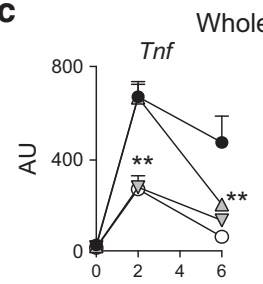

Whole lung
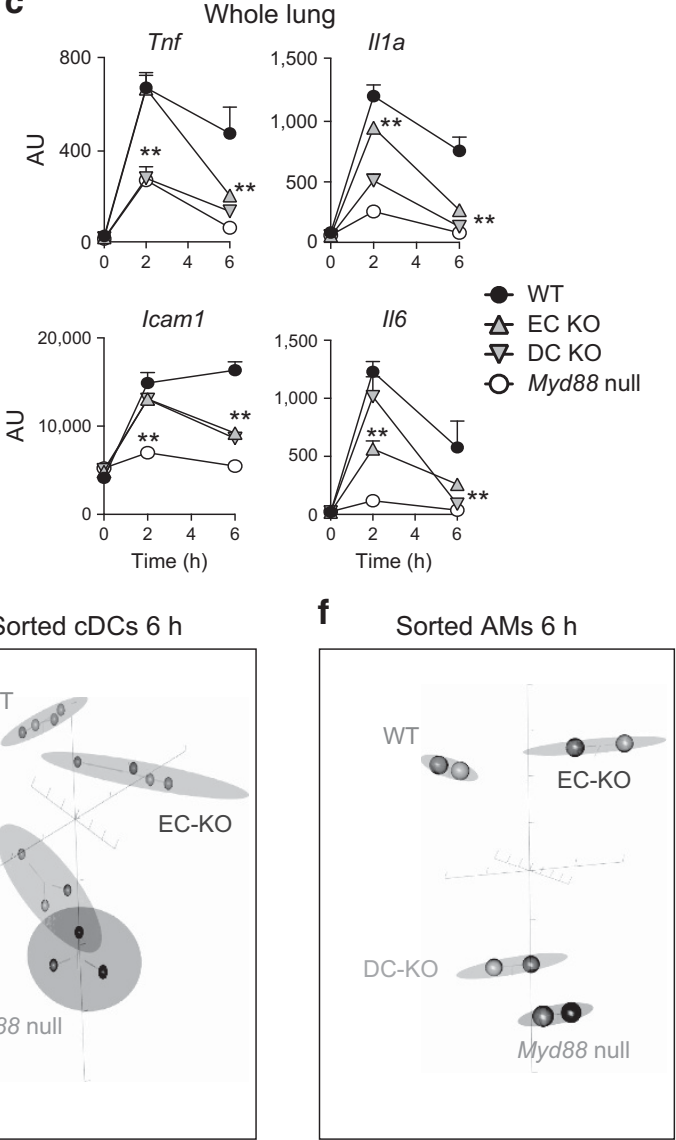

d $\mathrm{AM}$ and $\mathrm{CDC}$ gating:

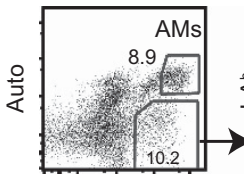

CD11c

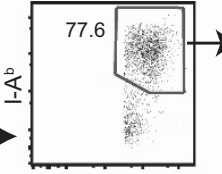

CD11c

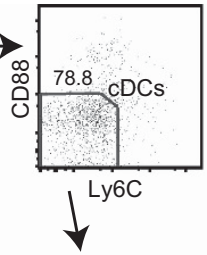

Post-sort of cDCs

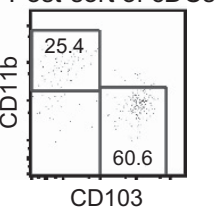

e

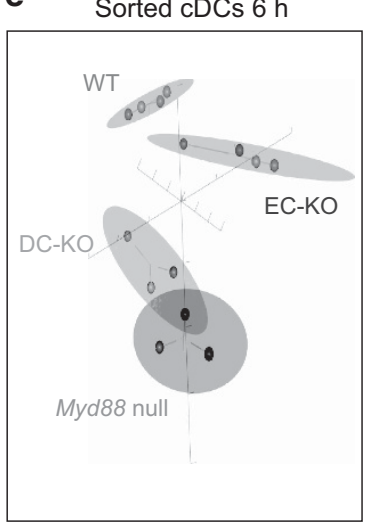

i

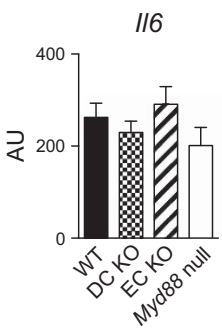

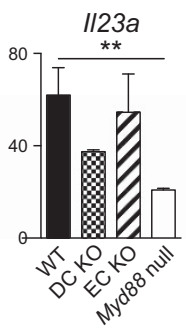

Sorted cDCs $6 \mathrm{~h}$ Fkbp5
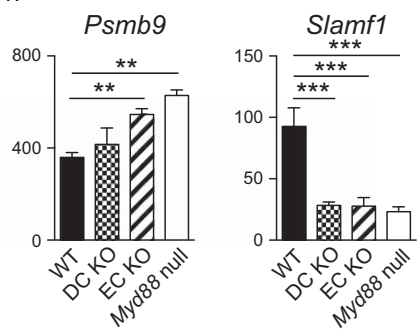

h

j

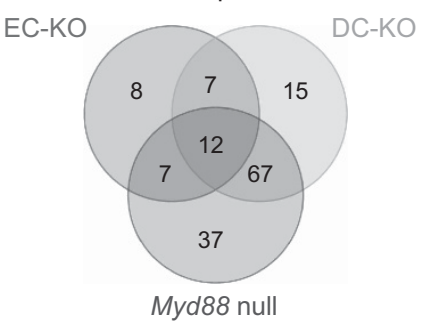

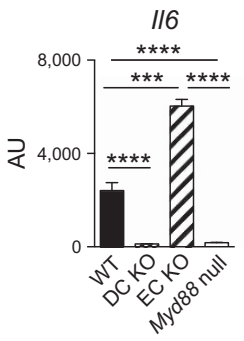

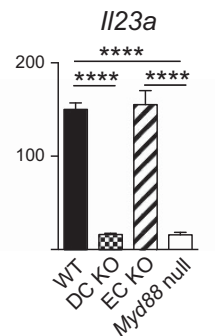

Sorted AMs $6 \mathrm{~h}$

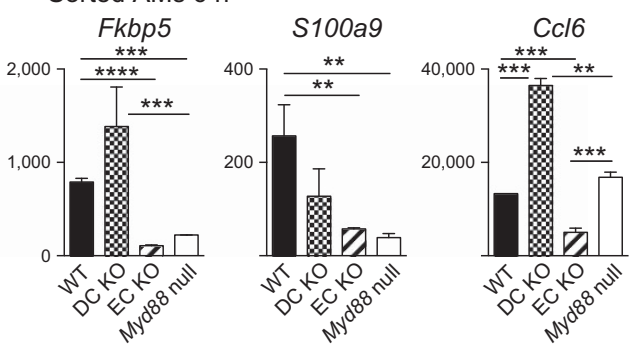

Figure 4 Immune gene expression in whole lung, $\mathrm{CDCs}$ and $\mathrm{AMs}$ at $6 \mathrm{~h}$ post-sensitization. (a) BGA plot of immune gene expression in whole lung at 0 and $6 \mathrm{~h}$ after OVA/FLA-mediated allergic sensitization of the indicated mouse strains. (b) Venn diagram showing the number of genes differentially expressed between lungs of WT mice (reference group) and lungs of the indicated mouse strains at $6 \mathrm{~h}$ post-sensitization ( $\geqslant 1.5 \mathrm{fold} ; P \leqslant 0.01, \mathrm{MTI} \geqslant 32$ for at least one group). (c) Time course of lung gene expression in the indicated strains. ${ }^{\star \star} P \leqslant 0.01$ as compared to WT reference strain. (d) Gating strategy for purification of AMs and cDCs. (e,f) BGA plot of immune gene expression in $\mathrm{cDCs}(\mathbf{e})$ and AMs (f) sorted from lungs of the indicated mouse strains $6 \mathrm{~h}$ after OVA/FLA-mediated sensitization. ( $\mathbf{g}, \mathbf{h})$ Venn diagrams showing the number of immune genes differentially expressed ( $\geqslant 1.5$ fold; $P \leqslant 0.01$, $\mathrm{MTI} \geqslant 32$ for at least one group) in $\mathrm{CDCs}(\mathbf{g})$ and AMs (h) of the indicated strains of mice compared to reference WT mice. (i,j) Relative expression of the indicated genes in purified $\mathrm{CDCs}$ (i) and AMs (j) of the indicated mouse strains at $6 \mathrm{~h}$ post-sensitization. For purified $\mathrm{AMs}$ and $\mathrm{cDCs}$, lungs from three mice were pooled prior to sorting each cell sample. $n=3-4$ (cDCs) and $n=2$ (AMs). ${ }^{* *} P<0.01,{ }^{\star \star *} P<0.001,{ }^{* * \star *} P<0.0001$. AM, alveolar macrophage; BGA, Between group analyses; CDC, conventional dendritic cells; FLA, flagellin; OVA, ovalbumin; WT, wild type. A full color version of this figure is available at the Mucosal Immunology journal online. 
and Illa (Figure 4c), the latter being important for Th17 differentiation. ${ }^{26}$ In addition, several genes were identified whose expression in whole lung was affected by MyD88 deletion in both ECs and Cd11c-expressing cells, including Il6, which is also important for Th17 differentiation, ${ }^{27}$ and the cell adhesion molecule, Icam1. Because the Cd11c-cre transgene is expressed in AMs as well as DCs, we separately purified these cells types from mice $6 \mathrm{~h}$ after OVA/FLA-mediated allergic sensitization (Figure 4d). cDCs from DC-KO mice and Myd88 null mice had similar gene expression profiles, but differed from the profiles of cDCs from WT and EC-KO mice (Figures 4e and g and Supplementary Table 6). Similar results were obtained when expression in AMs was studied (Figures $4 \mathbf{f}$ and $\mathbf{h}$ and Supplementary Table 7). These results confirm that OVA/ FLA-induced immune gene expression in cDCs and AMs is largely controlled by cell-intrinsic MyD88 signaling. Several cytokine encoding genes, including Illa, Il6 and Il23a, were much more highly expressed in AMs than in cDCs or ECs (Figures 4i and $\mathbf{j}$ and Supplementary Figure $2 \mathbf{b}$ ), suggesting that through their production of IL- $1 \alpha$, IL- 6 and IL-23, AMs may cooperate with cDCs to promote Th17 differentiation.

Our observation that Myd88 expression in ECs contributes to Th2 development and eosinophilic inflammation suggested that there might be crosstalk between ECs and DCs. To test this, we compared gene expression in CDCs of WT and EC-KO mice. We identified multiple immune genes whose expression in cDCs was partly controlled by MyD88 signaling in ECs, including Fkbp5, Psmb9, and Slamf1 (Figure $\mathbf{4 i}$ and Supplementary Table 6). Expression of some genes in AMs was also controlled by MyD88 signaling in ECs. For example, Fkbp5, S100a9, and Ccl6 were all decreased in AMs of EC-KO mice, whereas Il6 was increased in these cells (Figure $\mathbf{4 j}$ and Supplementary Table 7). Together, these data show that during allergic sensitization, expression of some immune genes in $\mathrm{CDCs}$ and AMs is controlled in part by signals they receive from ECs.

\section{MyD88 controls chromatin accessibility at select promoters in $\mathrm{CDCs}$}

To gain additional insight into how MyD88 controls gene expression in lung cDCs, we examined the impact of cellspecific MyD88 signaling on chromatin accessibility in cDCs using ATAC-seq, an assay based on the ability of transposases to introduce DNA sequencing adaptors into open chromatin. ${ }^{28}$ As expected, 'peaks' corresponding to multiple overlapping sequencing reads at open chromatin mapped to transcription start sites and enhancer regions, as well as intergenic and intronic regions (Figure 5a and Supplementary Table 8). Patterns of peak distribution among these categories were similar for all genotypes examined (data not shown). Restricting our analyses to chromatin at promoter regions defined as being within 500 base pairs (bp) of known transcription start sites, we identified 227 genes whose chromatin accessibility differed ( $\geqslant 1.3$-fold, $P \leqslant 0.05$, at least one peak $\geqslant 100$ reads) between WT and DC-KO mice $6 \mathrm{~h}$ after allergic sensitization (Figure $5 \mathbf{b}$, top left). Of these, 85 genes were also different between these same genotypes at steady state $(0 \mathrm{~h})$, whereas 142 loci were different only after sensitization $(6 \mathrm{~h})$. We identified an additional 60 genes whose chromatin accessibility in lung cDCs at $6 \mathrm{~h}$ was different between WT and EC-KO mice (Figure $5 \mathbf{b}$, top right), suggesting that signals from ECs can also affect chromatin accessibility at some loci in cDCs.

To better understand how MyD88-dependent changes in chromatin accessibility affect gene expression, we used transcriptome wide arrays to study expression of genes that mapped to loci whose ATAC-seq peak sizes were dependent on Myd88 expression. Almost half of the genes whose chromatin differed between CDCs of WT and DC-KO mice at both 0 and $6 \mathrm{~h}$ were expressed, that is, above the minimum threshold intensity (see Supplementary Methods). Of these genes, 15 were differentially expressed ( $\geqslant 1.5$-fold) between $\mathrm{cDCs}$ of WT and DC-KO mice at baseline but not $6 \mathrm{~h}$. Another 19 genes were induced, that is, differentially expressed at $6 \mathrm{~h}$ (Figure $5 \mathrm{~b}$, bottom left, and Supplementary Table 9a). By contrast, the majority of genes whose genotype-specific differences in chromatin accessibility were only apparent at $6 \mathrm{~h}$, but not $0 \mathrm{~h}$, did not yet display transcriptional differences between cDCs of WT and DC-KO mice (Supplementary Table 9b). Very few genes whose chromatin accessibility in cDCs was different between WT and EC-KO mice were expressed above the minimum threshold intensity (Figure 5b, bottom right, and Supplementary Table 9c).

\section{Cell-intrinsic MyD88 controls gene expression through multiple mechanisms}

Transcriptome-wide arrays have multiple probes for each gene, allowing simultaneous quantification of the $5^{\prime}$ and $3^{\prime}$ regions of RNA. Using this platform to measure RNAs in $\mathrm{cDCs}$ from the various strains (see Supplementary Methods), we identified two major types of MyD88-dependent gene induction that occurred during allergic sensitization. In the first, the ratio of gene expression between WT and DC-KO mice measured using $5^{\prime}$ probes was similar to ratios obtained using $3^{\prime}$ probes, consistent with regulatory control at the level of transcriptional initiation (see Supplementary Methods). In the second, the ratio of gene expression in WT and DC-KO mice was higher when measured using $3^{\prime}$ probes than with $5^{\prime}$ probes. The latter pattern is consistent with an indirect role for MyD88 in RNA elongation. Approximately one-third of the genes induced in cDCs by allergic sensitization met the criteria to be included in this category (Supplementary Table 9a). As an illustrative example, chromatin at the $C c r 7$ locus was found to be more accessible in cDCs of WT mice than in those of either DC-KO or Myd88 null mice, both at 0 and $6 \mathrm{~h}$ (Figure 5c, left). Despite these differences in chromatin accessibility, at $0 \mathrm{~h}$ there were no apparent differences in $C c r 7$ mRNA levels among the various genotypes (Figure 5c, middle). However, after allergic sensitization, $C c r 7$ mRNA levels were markedly higher in cDCs of WT and EC-KO mice than in those of DC-KO and Myd88 null animals. Furthermore, the ratio of $\mathrm{Ccr} 7$ expression in DCs of WT mice compared to those of DC-KO mice was 
a

ATAC-seq differential peak distribution

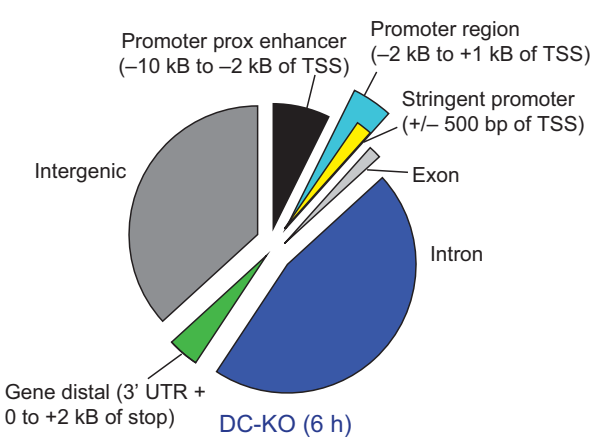

b

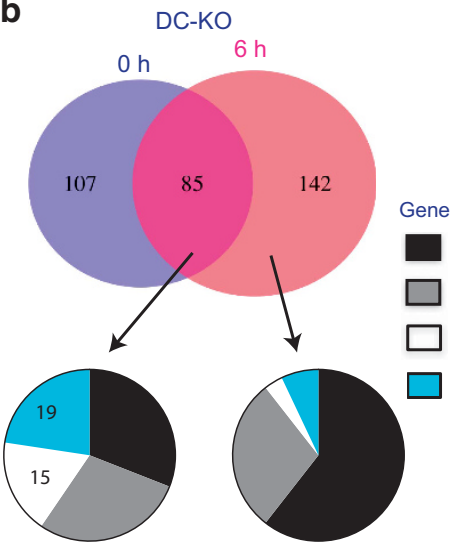

EC-KO

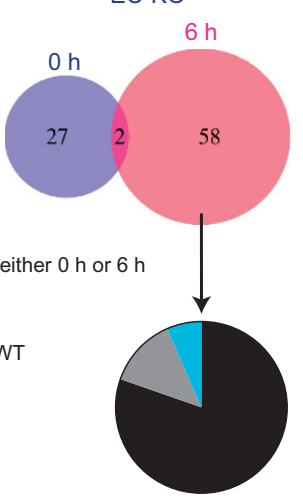

C
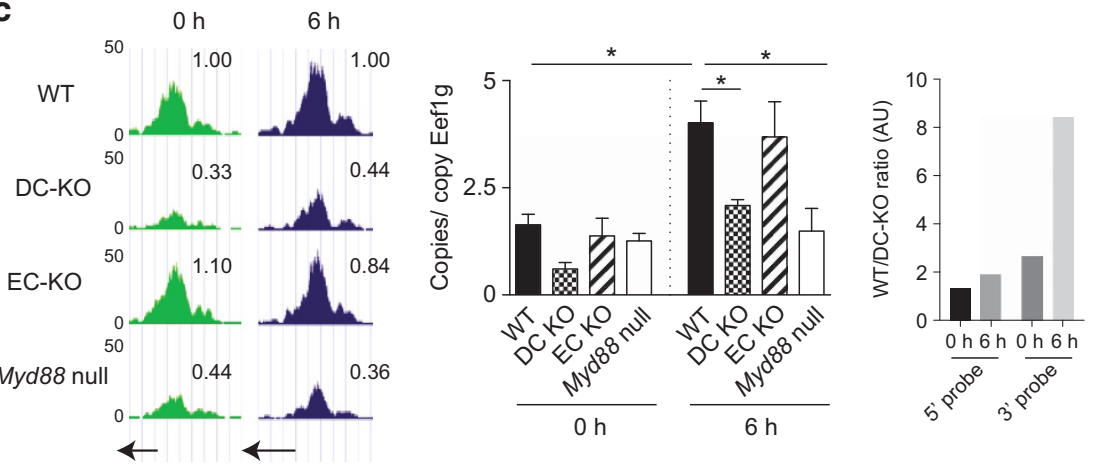

d

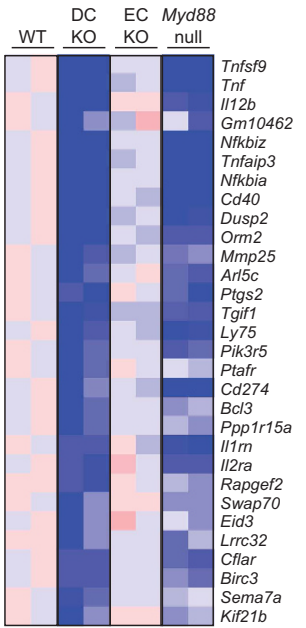

DC EC Myd88

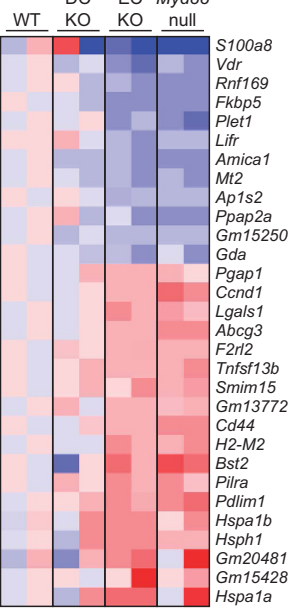

e
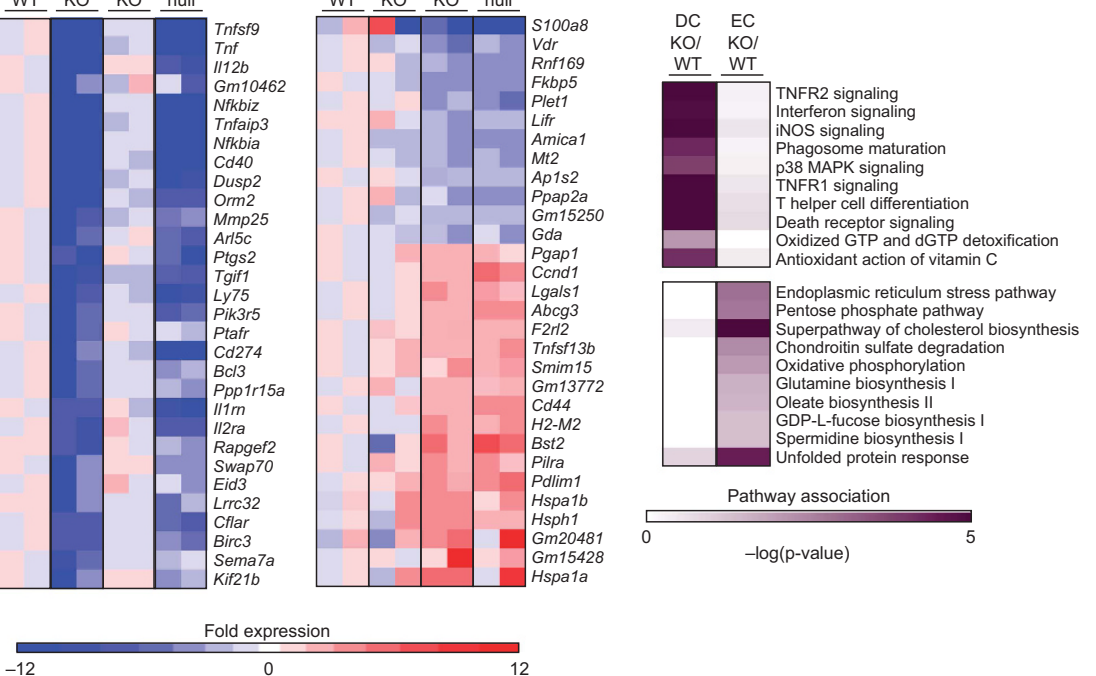

expression categories

Below MTI

Not different from WT at either $0 \mathrm{~h}$ or $6 \mathrm{~h}$

Different from WT at $0 \mathrm{~h}$

Greater difference from WT at $6 \mathrm{~h}$ than at $0 \mathrm{~h}$

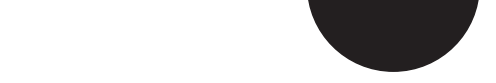


greater when expression was measured using a $3^{\prime}$ probe than with a $5^{\prime}$ probe (Figure 5c, right), suggestive of transcriptional control at the level of RNA elongation. Thus, MyD88 controls gene expression through multiple, distinct mechanisms. For Ccr7 and several other genes, MyD88 maintains open chromatin without affecting baseline gene expression, and during allergic sensitization promotes efficient RNA elongation.

\section{MyD88 signaling in DCs and ECs control distinct cellular processes in cDCs}

Analysis of gene expression in cDCs across the genome, without regard to ATAC-seq status, identified approximately 250 genes whose expression in cDCs was dependent on cell-intrinsic MyD88 signaling (Supplementary Table 10). Many of these genes have known roles in immunity and include Tnf, Il12b, and $C d 40$, as well as genes that regulate canonical NF- $\kappa b$ signaling, such as Tnfaip3 (which encodes A20), Nfkbiz, and Nfkbia (Figure 5d, left). Ingenuity Pathway Analysis confirmed that these genes contribute to immune signaling pathways (Figure 5e, top). We also identified genes whose expression in lung cDCs was controlled indirectly, through MyD88 signaling in ECs. Approximately half of these genes were negatively regulated by MyD88 signaling in ECs (Supplementary Table 10), and included $H 2-m 2$; Cd44; the heat shock protein genes, Hspala, Hspa1b, and Hsph1; the cell cycle and DNA damage sensing proteins, Ccnd1 and Ddit3. A different set of genes in cDCs was positively regulated by MyD88 signaling in ECs, including S100a8, S100a9, Fkbp5, and Amical (Figure 5d, right). Amical encodes junctional adhesion molecule-like protein, ${ }^{29}$ binding partner of the epithelial tight junction protein, coxsackie-adenovirus receptor. Interestingly, Ingenuity Pathway analysis revealed that MyD88 signaling in ECs affected pathways in cDCs that are associated with endoplasmic reticulum stress, oxidative phosphorylation, and cholesterol biosynthesis (Figure 5e, bottom). This suggests that ECs may indirectly affect the ability of cDCs to sense cellular stress and thereby modulate the downstream immune response.

\section{Effects of cell-specific Myd88 expression on DC phenotype and function}

We next examined the consequences of cell-intrinsic and extrinsic MyD88 signaling on DC phenotype and function. Following allergic sensitization, allergen-bearing cDCs increase their display of co-stimulatory molecules and migrate from the lung to the regional mediastinal (m)LNs. At $16 \mathrm{~h}$ postsensitization with OVA/FLA, we noted that CD40 was markedly reduced on DCs of DC-KO mice, and CD80 was modestly reduced on DCs of both DC-KO mice and EC-KO mice (Figure 6a), in agreement with the observed $C d 40$ and Cd80 transcriptional differences at $6 \mathrm{~h}$ (Supplementary Resource). We next studied the effect of cell-specific expression of Myd88 on DC trafficking by labeling the DCs with the fluorescent dye, $\mathrm{PKH}$ and inducing their migration with OVA/FLA. Analysis of mLNs $24 \mathrm{~h}$ after OVA/FLA instillation revealed fewer $\mathrm{PKH}+$ migratory DCs in mLNs of DC-KO mice than in those of WT mice (Figure $6 \mathbf{b}$ ). There was also a trend towards fewer migratory DCs in EC-KO mice, but it was not statistically significant. To investigate how these and other changes related to Myd88 expression in Cd11cexpressing cells and ECs affects early T helper cell development in regional LNs, we adoptively transferred OVA-specific, naïve $\mathrm{CD}^{+}{ }^{+} \mathrm{T}$ cells to recipient animals and sensitized them on a single occasion with OVA/FLA. mLNs of mice were excised from the sensitized mice and these mLN cells were cultured with OVA to stimulate cytokine production. As expected, Myd88 null mice had markedly lower amounts of type 2 cytokines and of IL-17A than did their WT counterparts (Figure 7a). IL-17A was also reduced in mLNs from DC-KO mice, but not in mLNs of EC-KO mice. Type 2 cytokines were similar in WT, DC-KO, and EC-KO mice after a single sensitization, but after two sensitizations, $\mathrm{mLN}$ of EC-KO mice had significantly lower amounts of IL-5 and IL-13 than did mLNs of WT mice (Figure $7 \mathbf{b}$ ). These data suggest that Cd11c-MyD88 mediated effects on Th17 differentiation are direct, and that EC-MyD88 dependent effects on Th2 polarization are indirect, possibly requiring another cell type. To investigate this, we separately purified $C d 11 c$-expressing $\mathrm{AMs}$ and $\mathrm{cDCs}$ from the lung at $6 \mathrm{~h}$ post sensitization with OVA/FLA, and tested their abilities to promote proliferation of naïve OT-II $\mathrm{T}$ cells and stimulate their production of cytokines. We found that $\mathrm{cDCs}$, but not AMs, could promote antigen-specific T-cell proliferation and production of type 2 cytokines and IFN $\gamma$, whereas only very small amounts of IL-17 were produced. Surprisingly, purified cDCs from each of the strains displayed similar capacities to stimulate $\mathrm{T}$ cells (Figure 7c). Although AMs on their own were incapable of stimulating $\mathrm{T}$ proliferation or cytokine production, we nonetheless investigated the impact of adding them to the cDC: T-cell co-cultures. No effect on T-cell proliferation was seen, but AMs from WT mice and EC-KO mice markedly reduced type 2 cytokine production by $\mathrm{T}$ cells, whereas AMs from Myd88 null mice DC-KO mice did not. AMs did not affect Th1 differentiation, as inferred from IFN $\gamma$ production. These data suggest that at least in this ex vivo experimental system, AMs produce one or more factors that selectively block Th2 differentiation in a manner dependent on MyD88 signaling in Cd11c-expressing cells.

\section{Effects of cell-specific Myd88 expression on T helper subsets in the lung}

The reduced amounts of IL-17 in mLNs of DC-KO mice, as well as their decrements in neutrophilic inflammation, suggested that Th17 cells in the lungs of these animals might be reduced. The reduction in type 2 cytokines in mLNs of EC-KO mice following a second sensitization with OVA/FLA and the decreased eosinophilia in lungs of these mice suggested that Th2 cells in the lung might be similarly reduced. To test this, we performed intracellular staining for IL-4, IL-5, IL-13 and IL-17A in CD4 $+\mathrm{T}$ cells sorted from lungs 3 days after the second OVA/FLA sensitization (day 10). Compared with WT 
a

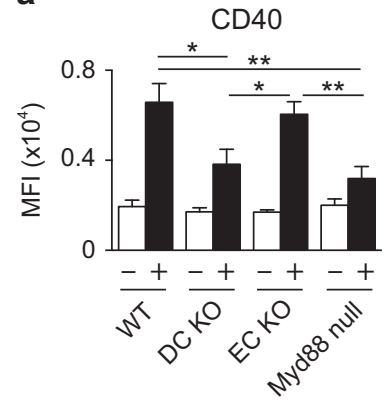

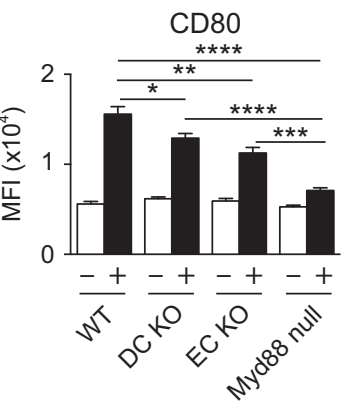

b

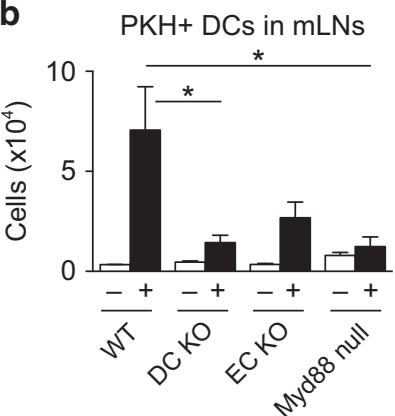

Figure 6 DC costimulatory molecule expression and DC migration to $\mathrm{mLNs}$ are regulated by MyD88. (a) Display of the costimulatory molecules, CD40 and CD80 on lung DCs (autofluorescence ${ }^{-}$CD11 $\mathrm{c}^{+} \mathrm{I}-\mathrm{A}^{\mathrm{b}} \mathrm{hi}$ ) from untreated mice $(-)$ and $16 \mathrm{~h}$ after OVA/FLA instillation ( + ). Mean fluorescence intensity (MFI). Data are representative of four experiments with 4-6 mice per group. (b) Migration of PKH-labeled DCs from the lung to $\mathrm{mLNs}$ at baseline (-) and $24 \mathrm{~h}$ after OVA/FLA instillation ( + ). Data are representative of three experiments with 3-6 mice per group. ${ }^{*} P<0.05,{ }^{* \star} P<0.01,{ }^{* * *} P<0.001$, ${ }^{* \star \star \star} P<0.0001$. DC, dendritic cells; FLA, flagellin; OVA, ovalbumin.

mice, DC-KO mice and Myd88 null mice had fewer Th17 cells in the lung, whereas no reduction was seen in EC-KO mice (Figure 7d). Conversely, intracellular staining for IL-4, -5 and -13 revealed that EC-KO mice had fewer Th2 cells compared with WT mice, whereas no reduction in Th2 cells was seen with DC-KO mice. Together, these data suggest that MyD88 signaling in Cd11c-expressing cells promotes Th17 development and consequent neutrophilic inflammation, whereas MyD88 signaling in ECs favors Th2 development and eosinophilic inflammation.

\section{DISCUSSION}

It is well established that MyD88 signaling is critical for innate immune responses to a wide variety of stimuli, including inhaled allergens. However, the extent to which MyD88 signaling in different cell types in the lung contributes to asthma is poorly understood. Here, we found using two different models of asthma that MyD88 expression in ECs impacts gene expression in cDCs and also profoundly affects immune responses. Specifically, MyD88 expression in ECs was found to be required for robust eosinophilia, whereas MyD88 expression in Cd11c-expressing cells was required for strong neutrophilic responses. This suggests that inhibiting MyD88 function in only one of these cell types might ameliorate disease features in individuals that display predominantly neutrophilic or eosinophilic asthma. Our current findings are consistent with studies showing that Tlr4 expression in non-hematopoietic cells contributes to eosinophilia ${ }^{15,30}$ and that Tlr4 expression in hematopoietic cells promotes airway neutrophilia in mouse models of asthma ${ }^{30}$ and further identifies ECs and Cd11c-expressing cells as requiring $M y d 88$ expression for the development of eosinophilic and neutrophilic inflammation, respectively.

MyD88 signaling in Cd11c-expressing cells was required for robust expression of $\mathrm{Ccr} 7$, and in agreement with this, DC migration from the lung to regional LNs was reduced in DC$\mathrm{KO}$ mice. This likely contributed to the reduced amounts of IL17 in lung-draining LNs of DC-KO mice, and consequently reduced numbers of Th17 cells and neutrophils in their lungs after allergen challenge. Although $\mathrm{C} c r 7$ expression was not reduced in EC-KO mice, they had a trend towards fewer migratory DCs in regional LNs. Ccr7 is required for DC migration, but other genes can also contribute. ${ }^{31,32}$ It is possible that altered gene expression in ECs can directly impact DC migration by affecting DC: EC adhesion, or indirectly affect migration by modifying gene expression in DCs.

MyD88 signaling in Cd11c-expressing cells was required for robust expression in cDCs of the Th17-promoting cytokines, Il6 and Il23a, and also for their display of CD40 which contributes to Th17 development. ${ }^{33,34}$ While these changes are consistent with reduced numbers of Th17 cells and airway neutrophils in DC-KO mice, our finding that AMs have higher expression of Illa, Il6 and Il23a than do cDCs suggested that AMs might also have a role in directing Th17 differentiation. On their own, AMs failed to activate $\mathrm{T}$ cells, and when added to DC: T-cell cultures had a minimal effect on IL-17 production, possibly because the cultures lacked a cell type or cytokine necessary for robust Th17 differentiation. The apparent discrepancy between this result and our observation that Myd88 null mice do not produce detectable amounts of cytokines in LNs might be partly due to the fact that equivalent numbers of $\mathrm{cDCs}$ were added to the ex vivo co-cultures, which would circumvent the DC migration defect in Myd88 null mice. Also, cDCs collected from the lung at $6 \mathrm{~h}$ post-sensitization have not undergone the MyD88-dependent increases in co-stimulatory molecules to the extent that are seen in DCs arriving in regional LNs. Interestingly, when added to the cDC: T-cell cultures, AMs selectively inhibited type 2 cytokine production in a manner dependent on Myd88. Whether this effect of AMs on T cells in vitro is relevant to $\mathrm{T}$-cell differentiation in vivo is unclear as AMs are non-migratory and therefore unable to traffic to regional LNs where most effector T-cell differentiation is thought to occur. Naïve T cells primarily reside in the blood and lymphatic system, but some of them also enter non-lymphoid organs, including the lung, ${ }^{33,35}$ and mice whose DCs cannot migrate to regional LNs can nonetheless develop strong responses to allergens. ${ }^{33,36}$ Thus, it is possible that naïve $\mathrm{T}$ 
a

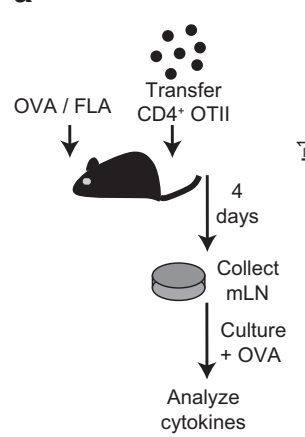

Single sensitization
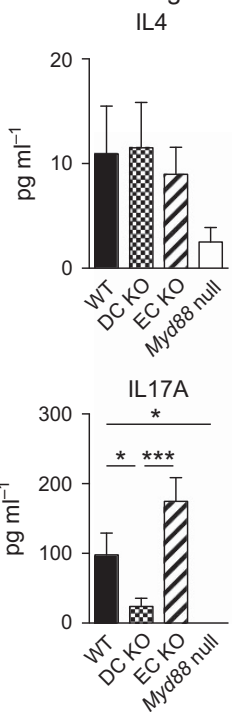

C
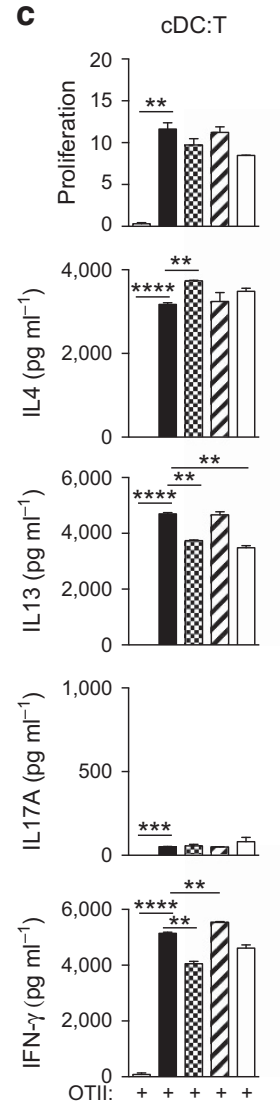

APC genotype: - 2 은에
AM:T
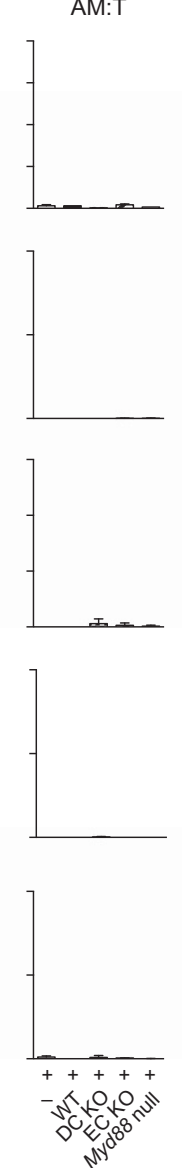
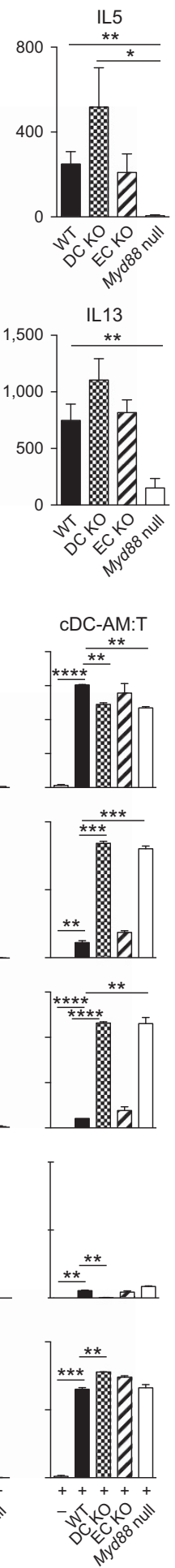

b

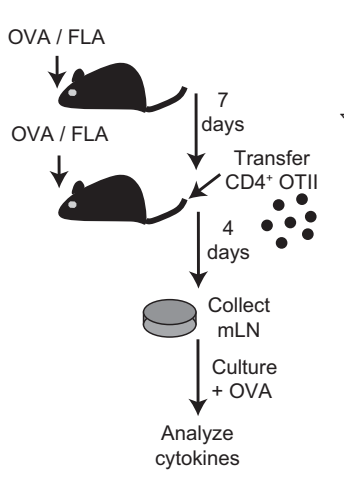

Two sensitizations
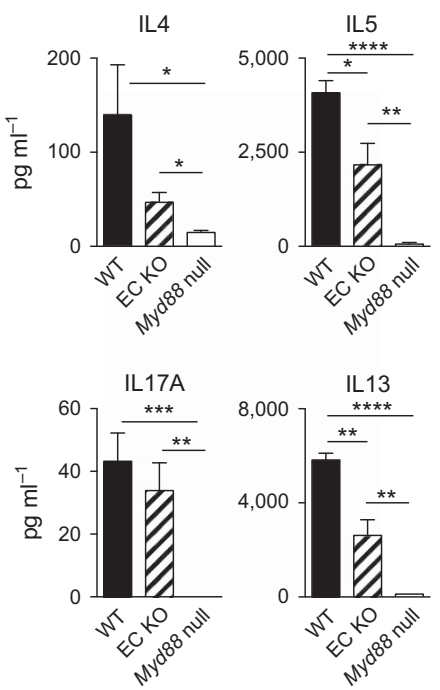

d
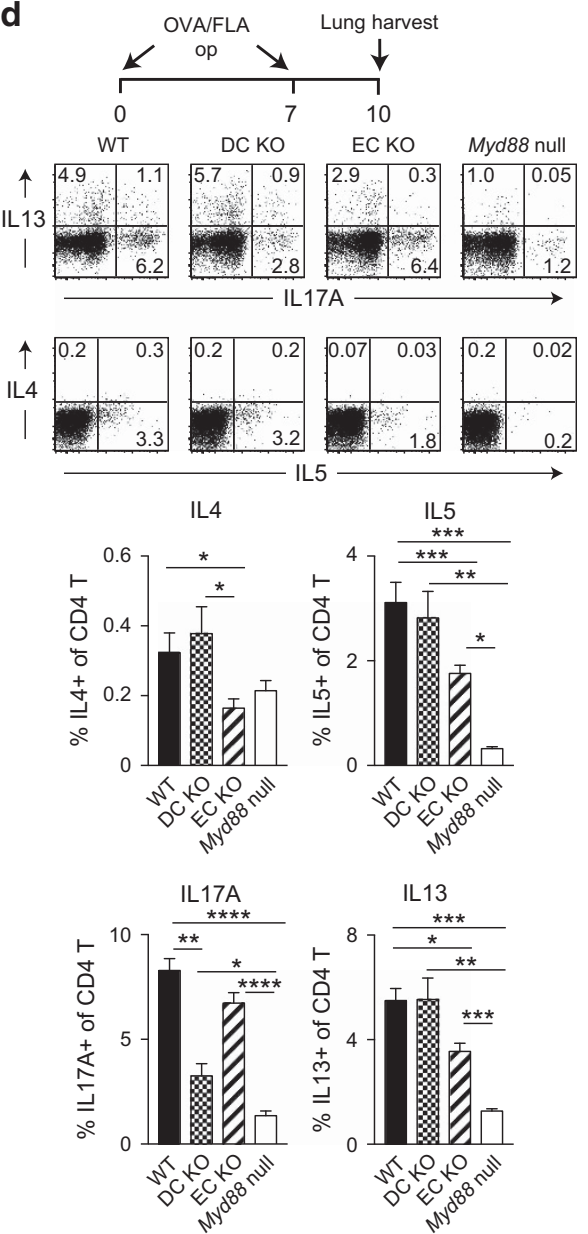

Figure 7 Differential control of Th2 and Th17 cytokine production by EC and DC expression of Myd88 (a,b) Cytokines in mLNs after a single sensitization (a) or two sensitizations (b) with $100 \mu \mathrm{g}$ OVA and $1.25 \mu \mathrm{g} \mathrm{FLA}$. (c) T-cell proliferation and cytokine production in co-cultures of OT-II T cells mixed with the indicated APC(s) sorted from lungs of mice $6 \mathrm{~h}$ after sensitization with OVA/FLA. (d) Intracellular staining for IL-17 and type 2 cytokines in $\mathrm{CD}_{4}^{+} \mathrm{T}$ cells of mice sensitized with OVA/FLA o.p. on days 0 and 7 and collected on day 10. Lung cells were stimulated with Phorbol 12-myristate 13-acetate (PMA) and ionomycin prior to staining. Data shown are (a) representative of two experiments with 8-10 mice per group; (b) from a single experiment, representative of two, 4-5 mice per group; (c) representative of a single experiment performed with duplicate samples; and (d) from a single experiment with 3-4 mice per genotype. ${ }^{\star} P<0.05$, ${ }^{\star *} P<0.01$, ${ }^{\star * \star} P<0.001$, ${ }^{\star * * \star} P<0.0001$. APC, antigen presenting cell; DC, dendritic cells; EC, epithelial cells; FLA, flagellin; IL, interleukin; mLN, mediastinal lymph node; o.p., oropharyngeal; OVA, ovalbumin. 
cells can undergo differentiation to Th17 cells in the lung itself, where their differentiation may be influenced by AM-derived cytokines.

Owing to their position on the luminal surface of the entire respiratory tree, ECs are exposed to a wide variety of antigens and potentially damaging environmental insults. We have shown that the expression of many genes in ECs is dependent on MyD88 signaling in those cells, including genes expected to affect antigen presenting cell (APC) function. These genes included Csf2, which encodes Granulocyte Macrophage Colony Stimulating Factor (GM-CSF), a potent activator of AMs and $\mathrm{DCs},{ }^{33,37}$ and $s 100 a 8 / a 9$, which encodes proteins that bind TLR4 and activate DCs. ${ }^{33,38}$ Previous studies have suggested roles for other EC-derived factors in shaping responses to inhaled allergens, including ATP, uric acid, IL-25, IL-33, and thymic stromal lymphopoietin (TSLP). ${ }^{33,39}$ We did not detect mRNA for Il25 or Tslp in sorted ECs, although low amounts of Tslp were seen in whole lung, in agreement with a previous report that ECs are not a major source of this cytokine. ${ }^{40}$ By contrast, Il33 mRNA was detected at relatively high levels in sorted ECs, consistent with the previous observation that alveolar type II ECs are the major pulmonary source of IL$33{ }^{33,41} \mathrm{It}$ is also possible that allergen inhalation triggers release of pre-formed IL-33. Whether that cytokine directly activates lung DCs is unclear, however, because Myd88 expression in DCs was not required for the adjuvant activity of IL-33.

RNA profiling and analysis of chromatin accessibility by ATAC-seq revealed control of gene expression in cDCs by both cell-intrinsic and -extrinsic MyD88 signaling. We unexpectedly found that chromatin accessibility at many promoter regions is established in a MyD88-dependent manner even prior to allergic sensitization. The ligand/receptor interactions that engage MyD88 to maintain an open chromatin at these loci during steady state are unknown. It is possible that low amounts of microbial products in the lung might signal through one or more TLRs to maintain a basal level of MyD88 signaling. Alternatively, constitutive production of endogenous cytokines, such as IL-1 or IL-33, which also signal through MyD88, might sustain open chromatin at these loci. How this MyD88 signaling leads to chromatin opening is also unknown, but might involve the induction of pioneer transcription factors (TFs), so named because of their ability to bind and open closed chromatin. ${ }^{42}$ Additional studies will be required to test this hypothesis and to determine whether cell-intrinsic and extrinsic MyD88 signaling trigger chromatin binding by different pioneer TFs.

Despite their open chromatin at baseline, many genes were not strongly expressed until after OVA/FLA-mediated allergic sensitization. This suggests that for these genes, MyD88 is not only required to open the chromatin at steady state, but also required for responses to a second, activating signal. For many genes, the ratio of expression of WT to DC-KO mice after sensitization was higher when measured using $3^{\prime}$ probes than with $5^{\prime}$ probes, suggesting that this second signal can act by promoting RNA elongation. This hypothesis is consistent with the known ability of RNA polymerase II (Pol II) to initially associate with an incomplete transcriptional complex and generate only short (20-65 nucleotide) RNA segments until receiving an activation signal required to generate full-length mRNA. ${ }^{43}$ This second step can be triggered by the binding of additional TFs and is associated with release of negative elongation factors from the complex through the positive transcription elongation factor $\mathrm{b}$ (P-TEFb kinase). It remains to be determined whether different TFs are involved in this putative elongation step than those that direct chromatin opening, and whether these TFs are differentially induced by cell-intrinsic and -extrinsic MyD88 signaling.

Ingenuity pathway analysis software revealed that MyD88 signaling in Cd11c-expressing cDCs primarily affected genes associated with the innate immune response and $\mathrm{CDC}$ effector function. Most of these genes are likely controlled by MyD88 signaling in the cDCs themselves, but because $\mathrm{Cd11c}$-cre is also expressed in AMs, it is also possible that MyD88 deficiency in those cells indirectly affects gene expression in cDCs. Nonetheless, the genetic approach used here avoids the confounding problem of inefficient and variable depletion of AMs by irradiation in bone marrow chimeric mice ${ }^{16}$ and provides a good model to evaluate the role of Myd88 in these cells. Although IL- $1 \alpha$ can be produced by bronchial ECs, ${ }^{44}$ our data suggest that the primary MyD88-dependent cell source in the lung is the AM. Interestingly, the loss of MyD88 in ECs was associated with increased transcription in cDCs of genes involved in cellular stress and lipid metabolism, including several genes in the superpathway of cholesterol synthesis. Several intermediates in the cholesterol biosynthetic pathway function as ligands of ROR $\gamma \mathrm{t}$, the nuclear receptor critical for the development of Th17 cells, ${ }^{45}$ and blocking cholesterol synthesis impairs the differentiation of these cells. ${ }^{46}$ Furthermore, accumulation of intracellular cholesterol leads to increased Th17 responses, but decreased Th2 responses, in the lung. ${ }^{47}$ Whether transfer of lipids from DCs to T cells contributes to Th17 differentiation is not known. However, lipids and other material can be transferred from DCs to T cells by secreted exosomes, ${ }^{48}$ or through direct contact at the immunological synapse. ${ }^{49}$ It will be of interest to identify the MyD88-dependent signals from ECs that act on lung $\mathrm{cDCs}$ to control their cholesterol metabolism, and to determine if the altered cholesterol pathways in cDCs contributes to the increased Th17 cell differentiation seen in EC-KO mice.

Our finding that both cell-intrinsic and -extrinsic MyD88 signaling contributes to $\mathrm{CDC}$ reprogramming provides important insights into the cellular and molecular basis of allergic sensitization. Given the heterogeneity seen in clinical asthma and the differential responsiveness of patients to current therapies, these insights will be useful in the design of new therapies specifically tailored to target asthma that is predominantly eosinophilic or neutrophilic in nature.

\section{MATERIALS AND METHODS}

Mice. The following strains were purchased from Jackson Laboratory (Bar Harbor, ME): C57BL/6, OTII (C57BL/6-Tg(TcraTcrb)425Cbn/J), 
$M y d 88^{-/-}$(B6.129P2(SJL)-Myd88tm1.1Defr/J), Myd88 $8^{f x / f x}$ (B6.129P2 (SJL)-Myd88 $\left.{ }^{\text {tm1Defr/J }}\right)$, Cd11c-cre (B6.Cg-Tg(Itgax-cre)1-1Reiz/J), and

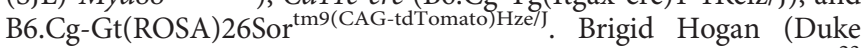
University) provided $\mathrm{B} 6 ; \mathrm{D} 2-\mathrm{Tg}(\mathrm{Sftpc}-\mathrm{cre}) 1 \mathrm{Blh}$ (Sftpc-cre) mice. ${ }^{23}$ $\mathrm{EC}-\mathrm{KO}$ mice and DC-KO mice were generated by crossing conditionally mutant $M y d 88^{f x / f x}$ mice $^{22}$ to Sftpc-cre mice and Itgax-cre mice, respectively. Mice were housed in specific pathogen-free conditions at the NIEHS and used between 6 and 12 weeks of age in accordance with guidelines provided by the Institutional Animal Care and Use Committees.

Processing frozen sections. Lungs were inflated with $4 \%$ paraformaldehyde, serially transferred into 15,20 , and then $30 \%$ sucrose, and finally into a 1:1 mixture of optimal cutting temperature (OCT) compound and $30 \%$ sucrose in phosphate-buffered saline (PBS). The lungs were then frozen on dry ice and sectioned using a cryostat.

Images of frozen sections. Tiled images of frozen sections were acquired at $\times 400$ magnification on an Automated Epifluorescence Microscope (Zeiss, Oberkochen, Germany) and assembled using Zen Blue software (Zeiss).

Images of precision cut lung slices. Lungs were inflated with $2 \%$ lowmelt agarose at $40^{\circ} \mathrm{C}$, and the upper right apical lung lobe was sliced into $150 \mu \mathrm{m}$ sections using a VF-300 Compresstome (Precisionary Instruments, Greenville, NC). Each tiled image was taken at $\times 200$ magnification on a 710 Confocal Microscope (Zeiss) and assembled using Zen Black software (Zeiss).

Mouse models of asthma. For the OVA/FLA model of asthma, mice were sensitized by oropharyngeal (o.p.) instillations of $100 \mu \mathrm{g}$ endotoxin-free OVA together with $1.25 \mu \mathrm{g}$ standard FLA from Salmonella typhimurium (InvivoGen, San Diego, CA) on days 0 and 7, then challenged on days 14,15 , and 16 with $1 \%$ OVA aerosol. (This FLA preparation signals through both TLR4 and TLR5 because it contains $10^{3}-10^{4}$ endotoxin units/mg protein.) In the HDM model of asthma, mice were sensitized with $1 \mu \mathrm{g} \mathrm{HDM}$ (Greer, Lenoir, NC) by o.p. instillation on days 0 and 7 and challenged by the same route on days 14,15 , and 16 . For both models, mice were collected at $4 \mathrm{~h}$ postchallenge to examine cytokines and chemokines, and at $24 \mathrm{~h}$ postchallenge to assess leukocyte recruitment to the airway. For the OVA/ IL-33 model of asthma, mice were sensitized by o.p. instillations of $100 \mu \mathrm{g}$ endotoxin-free OVA together with $500 \mathrm{ng}$ IL-33 (Biolegend, San Diego, CA) on days 0 and 7 , then challenged on days 14,15 , and 16 with $1 \%$ OVA aerosol.

Flow cytometry. Cells were blocked with anti-mouse CD16/CD32 (2.4G2) and normal mouse and rat serum (Jackson ImmunoResearch, West Grove, PA). For staining of surface antigens, cells were incubated with one or more of the following antibodies: CD3 (145-2C11), CD8 $\alpha$ (53-6.7), CD8 $\beta$ (53-5.8), CD19 (1D3), CD44 (IM7), IL-4 (11B11), IL-5 (TRFK5), IL-17A (TC11-18H10), Ly6C (AL-21), Ter119, (BD Biosciences, San Jose, CA); CD34 (MEC14.7), CD45 (104), CD88 (20/ 70), EpCAM/CD326 (G8.8) (Biolegend); B220 (RA3-6B2), CD25 (PC61.5), CD4 (GK1.5), CD11b (M1/70), CD11c (N418), CD31 (390), CD40 (1C10), CD49b (DX5), CD80 (16-10A1), CD103 (2E7), eF780 viability dye, I-A ${ }^{\mathrm{b}}$ (AF6-120.1), IL-13 (eBio13A), Ly6C/G (Gr-1) (RB68C5), SIRP-1 $\alpha /$ CD172a (P84), streptavidin eFluor450, 7-AAD (eBioscience, San Diego, CA). Lung DCs were examined for costimulatory molecules at baseline and $16 \mathrm{~h}$ after o.p. inhalation of OVA/ FLA.

Preparation of sorted ECs. Lungs were perfused, inflated, and digested with $4.5 \mathrm{U} \mathrm{ml}^{-1}$ elastase for $45 \mathrm{~min}$ at $37^{\circ} \mathrm{C}$. Minced lung was then digested with DNase for another $15 \mathrm{~min}$. Cells were strained through a $70 \mu \mathrm{m}$ filter, depleted for non-epithelial lineage markers (CD31, CD34, CD45) using AutoMACS column (Miltenyi Biotec, San Diego, CA). ECs were FACS sorted $\left(7-\mathrm{AAD}^{-} \mathrm{CD}^{-} 1^{-} \mathrm{CD} 34\right.$ $\mathrm{CD}_{4} 5^{-} \mathrm{EpCAM}^{+}$) with purity $>99 \%$.
Preparation of sorted cDCs and AMs. Lungs were perfused, minced, and digested in collagenase, DNAse, hyaluronidase, and Liberase TM for $1 \mathrm{~h}$. Single cells were isolated using a $70 \mu \mathrm{m}$ filter, and a $14.5 \%$ Nycodenz gradient was used to separate AMs and cDCs from other lung leukocytes. AMs were sorted for $\mathrm{CD}_{11 \mathrm{c}^{+}}$autofluorescence ${ }^{+}$, while cDCs were sorted for CD11c ${ }^{+}$autofluorescence ${ }^{-} \mathrm{I}^{-\mathrm{A}^{\mathrm{b}} \text { hi }} \mathrm{Ly6C}$ CD88 ${ }^{-}$by flow cytometry. Ly6C and CD88 were used to exclude inflammatory DCs and monocyte-derived (mo)DCs respectively. ${ }^{50}$ Purity was estimated at $>94 \%$ for AMs and cDCs.

RNA isolation from whole lung and sorted cells. Lungs were collected from mice at various times after oropharyngeal administration of $100 \mu \mathrm{g}$ OVA mixed with $1.25 \mu \mathrm{g}$ stFLA, and RNA was isolated using RNeasy kit (Qiagen, Germantown, MD). RNA from sorted cells was isolated by Trizol, followed by Qiagen RNeasy MinElute Cleanup Kit to remove any organic solvents.

Nanostring Mouse Immunology Gene Expression. RNA from whole lung and sorted ECs, cDCs, and AMs was hybridized to the Nanostring nCounter Mouse Immunology Gene Expression Codeset according to the manufacturer's instructions and quantified on the Nanostring nCounter machine (Nanostring, Seattle, WA). Only genes having a group mean intensity $\geqslant 32(\log 2 \geqslant 5)$ for at least one sample were analyzed (see Supplementary Materials and Methods).

Whole transcriptome microarray gene expression. RNA from sorted cDCs was processed and analyzed on the Affymetrix Mouse Whole Transcriptome Array 1.0 according to the manufacturer's instructions (Affymetrix, Santa Clara, CA). Only genes having a maximum mean bi-weight $\log 2$ probe signal of $\geqslant 6.0$ were considered above the minimum threshold intensity.

Pathway analysis. Pathway analysis was performed on whole transcriptome microarray data using the Ingenuity Pathway Analysis software (Redwood City, CA).

Library preparation for ATAC-seq. Following their isolation, cDCs were subjected to ATAC-seq as previously described ${ }^{28}$ with the following modifications: $25,000 \mathrm{cDCs}$ were lysed in cold lysis buffer (10 mm Tris- $\mathrm{HCl} \mathrm{pH} 7.5,10 \mathrm{~mm} \mathrm{NaCl}, 3 \mathrm{~mm} \mathrm{MgCl}_{2}, 0.1 \% \mathrm{NP}-40$ ) for $5 \mathrm{~min}$, and nuclei were incubated with $2.5 \mu \mathrm{l}$ of Tn5 Transposase in $25 \mu \mathrm{l}$ of reaction buffer. Purified DNA fragments were amplified with nine cycles of PCR, the PCR products purified by using AMPure XP beads (1:3 ratio of sample to beads, Beckman Coulter, Brea, CA), and the libraries sequenced on a NextSeq 500 (Illumina) in $50 \mathrm{bp}$ paired-end format.

$\mathbf{P K H}+$ DC migration from lung to $\mathbf{m L N}$. Mice were given $50 \mu \mathrm{l}$ of $10 \mu \mathrm{m}$ PKH26 oropharyngeally to label cells in vivo in the lung. Twenty-four hours later, mice were either not sensitized (baseline) or given OVA/FLA to sensitize the mice. After another $24 \mathrm{~h}$, the draining mediastinal lymph node was collected. Cells were isolated and stained for flow cytometry. $\mathrm{PKH}^{+}$DCs were identified based on staining for $\mathrm{PKH}$ as well as autofluorescent ${ }^{-} \mathrm{CD}_{11 \mathrm{c}^{+}}{\mathrm{I}-\mathrm{A}^{\mathrm{b}}+}^{+}$staining.

OT-Il cell transfer and draining lymph node cytokine measurement. Recipient mice were injected with $0.5-3 \times 10^{6} \mathrm{CD} 4^{+} \mathrm{T}$ cells from OTII mice, and sensitized 2-4 h later with o.p. instillation(s) of OVA/FLA. mLNs were collected 4 days later, and $5 \times 10^{6}$ cells per ml were cultured in cRPMI with $20 \mu \mathrm{g} \mathrm{ml}^{-1}$ OVA. Forty-eight hours later, supernatants were analyzed by ELISA for IL-4, IL-5, IL-13, IL-17A, and IFN- $\gamma$ according to the manufacturer's instructions.

DC-AM co-culture with naïve $\mathbf{T}$ cells. Mice were sensitized by instillation of OVA/FLA as described above, and $6 \mathrm{~h}$ later AMs and cDCs from three pooled mice were sorted separately for each genotype. Naïve $\mathrm{CD} 4^{+}$OTII cells were isolated from the spleens and lymph nodes of OTII mice and depleted using an antibody cocktail including $\mathrm{CD} 8 \alpha, \mathrm{CD} 8 \beta, \mathrm{CD} 11 \mathrm{~b}, \mathrm{CD} 11 \mathrm{c}, \mathrm{CD} 16 / 32$, CD19, CD25, B220, CD49b, I-A ${ }^{b}$ Ly6C/G, TER119, and CD44 on the AutoMACS Pro (Miltenyi 
Biotec, Auburn, CA). Naïve OTII cells $\left(5 \times 10^{4}\right.$ per well $)$ were cultured together with lung cDCs and/or AMs $\left(5 \times 10^{3}\right.$ per well) in a $5 \% \mathrm{CO}_{2}$ incubator in a U-bottom plate (BD Biosciences). After 5 days, $\mathrm{T}$ cells were counted to measure proliferation. T cells $\left(10^{5}\right.$ per well $)$ were then transferred to a 96-well flat bottom plate coated with $1 \mu \mathrm{g} \mathrm{ml}^{-1}$ anti CD3 and $5 \mu \mathrm{g} \mathrm{ml}^{-1}$ anti-CD28 antibodies (eBioscience). Twenty-four hours later, supernatants were analyzed by ELISA for IL-4, IL-13, IL-17A, and IFN- $\gamma$ according to the manufacturer's instructions

Intracellular cytokine staining of lung $T$ cells following two sensitizations. Mice were sensitized oropharyngeally with OVA/FLA on days 0 and 7. On day 10, lungs were collected, perfused, minced, and digested in collagenase, DNAse, hyaluronidase, and Liberase TM for $30 \mathrm{~min}$. Single cells were isolated using a $70 \mu \mathrm{m}$ filter, and a Histopaque-1083 gradient was used to separate the lymphocyte fraction. Cells were stimulated with phorbol 12-myristate 13-acetate (PMA) and ionomycin for $6 \mathrm{~h}$ and then cells were stained for extracellular markers, fixed and permeabilized, and stained for intracellular cytokines.

Statistical analysis. Unless stated otherwise, statistical significance in animal models of asthma were determined by Student's unpaired $t$-test. Statistical analysis of Nanostring, whole transcriptome array and ATAC-seq data are described in detail in the Supplementary methods.

SUPPLEMENTARY MATERIAL is linked to the online version of the paper at http://www.nature.com/mi

\section{ACKNOWLEDGMENTS}

We thank Maria Sifre and Carl Bortner for help with flow cytometry, Kevin Gerrish and Liwen Liu for assistance with microarray processing and data analysis advice, Rick Fannin and Esther Hou for help with Nanostring processing and preliminary data analysis, Ligon Perrow for support with animal experiments, Otis Lyght and Natasha Clayton for frozen tissue processing and sectioning, Gordon Flake for histological comparison of H\&E and fluorescence serial sections, Charles Tucker for assistance with confocal microscopy, Michael Sanderson and Jun Chen for guidance in processing and analyzing lung slices, John House for advice on pathway analysis, and Michael Fessler and Jennifer Martinez (NIEHS) for critical reading of the manuscript. This work was supported by the Intramural Research Program of the NIEHS (ZIA ES102025-09) to D.N.C. and (ES101965) to P.A.W.

\section{GEO ACCESSION NUMBER}

All Nanostring, microarray and ATAC-seq data have been deposited th the National Center for Biotechnology Information in the Gene Expression Omnibus repository under accession number: GSE79615. Gene expression data for whole lung at various timepoints, as well as sorted ECs at $2 \mathrm{~h}$, cDCs at $6 \mathrm{~h}$, and AMs at $6 \mathrm{~h}$ are included in an interactive display: $\mathrm{https}: / /$ jmw86069.github.io/myd88-asthma/.

\section{AUTHOR CONTRIBUTIONS}

S.Y.T, G.S.W, M.T, J.M.W, X.X., K.N., M.R.L.-C., H.N., K.M.G., P.A.W. performed experiments and/or analyzed the data. D.N.C. conceived of the project, and S.Y.T. and D.N.C. designed the experiments and wrote the paper.

\section{DISCLOSURE}

The authors declared no conflict of interest.

c) 2018 Society for Mucosal Immunology

\section{REFERENCES}

1. Moore, W.C. et al. Clinical heterogeneity in the severe asthma research program. Ann. Am. Thorac. Soc. 10 (Suppl), S118-S124 (2013).

2. Hastie, A.T. et al. Analyses of asthma severity phenotypes and inflammatory proteins in subjects stratified by sputum granulocytes. J. allergy clin. immunol. 125, 1028-1036 e1013 (2010).
3. Poon, A.H., Eidelman, D.H., Martin, J.G., Laprise, C. \& Hamid, Q. Pathogenesis of severe asthma. Clin. exp. allergy 42, 625-637 (2012).

4. Moore, W.C. et al. Sputum neutrophil counts are associated with more severe asthma phenotypes using cluster analysis. J. allergy Clin. immunol. 133, 1557-1563 e1555 (2014).

5. Chung, K.F. etal. International ERS/ATS guidelines on definition, evaluation and treatment of severe asthma. Eur. Respir. J. 43, 343-373 (2014).

6. Green, R.H., Brightling, C.E., Woltmann, G., Parker, D., Wardlaw, A.J. \& Pavord, I.D. Analysis of induced sputum in adults with asthma: identification of subgroup with isolated sputum neutrophilia and poor response to inhaled corticosteroids. Thorax 57, 875-879 (2002).

7. McKinley, L. et al. TH17 cells mediate steroid-resistant airway inflammation and airway hyperresponsiveness in mice. J. immunol. 181, 4089-4097 (2008).

8. Barczyk, A., Pierzchala, W. \& Sozanska, E. Interleukin-17 in sputum correlates with airway hyperresponsiveness to methacholine. Respir. Med. 97, 726-733 (2003).

9. Hellings, P.W. et al. Interleukin-17 orchestrates the granulocyte influx into airways after allergen inhalation in a mouse model of allergic asthma. Am. J. Respir. Cell Mol. Biol. 28, 42-50 (2003).

10. Kaminska, M. et al. Airway remodeling in subjects with severe asthma with or without chronic persistent airflow obstruction. J. allergy clin. immunol. 124, 45-51 e41-44 (2009).

11. Molet, S. et al. IL-17 is increased in asthmatic airways and induces human bronchial fibroblasts to produce cytokines. J. allergy clin. immunol. 108, 430-438 (2001).

12. Wilson, R.H., Whitehead, G.S., Nakano, H., Free, M.E., Kolls, J.K. \& Cook, D.N. Allergic sensitization through the airway primes Th17dependent neutrophilia and airway hyperresponsiveness. Am. j. respir. crit. care med. 180, 720-730 (2009).

13. Chesne, J., Braza, F., Mahay, G., Brouard, S., Aronica, M. \& Magnan, A. IL17 in severe asthma. Where do we stand?. Am. j. respir. crit. care med. 190, 1094-1101 (2014).

14. Lambrecht, B.N. \& Hammad, H. Lung dendritic cells in respiratory viral infection and asthma: from protection to immunopathology. Annu. rev. immunol. 30, 243-270 (2012).

15. Hammad, H., Chieppa, M., Perros, F., Willart, M.A., Germain, R.N. \& Lambecht, B.N. House dust mite allergen induces asthma via Toll-like receptor 4 triggering of airway structural cells. Nat. med. 15, 410-416 (2009).

16. Janssen, W.J. et al. Fas determines differential fates of resident and recruited macrophages during resolution of acute lung injury. Am. j. respir. crit. care med. 184, 547-560 (2011).

17. Citrin, D.E. et al. Role of type II pneumocyte senescence in radiationinduced lung fibrosis. J. Natl. Cancer Inst. 105, 1474-1484 (2013).

18. Piggott, D.A. et al. MyD88-dependent induction of allergic Th2 responses to intranasal antigen. J. clin. invest. 115, 459-467 (2005).

19. Wilson, R.H. et al. The Toll-like receptor 5 ligand flagellin promotes asthma by priming allergic responses to indoor allergens. Nat. med. 18, 1705-1710 (2012).

20. Caton, M.L., Smith-Raska, M.R. \& Reizis, B. Notch-RBP-J signaling controls the homeostasis of CD8- dendritic cells in the spleen. J. exp. med. 204, 1653-1664 (2007).

21. Schlitzer, A. et al. Identification of CDC1- and CDC2-committed DC progenitors reveals early lineage priming at the common DC progenitor stage in the bone marrow. Nat. immunol. 16, 718-728 (2015).

22. Hou, B., Reizis, B. \& DeFranco, A.L. Toll-like receptors activate innate and adaptive immunity by using dendritic cell-intrinsic and -extrinsic mechanisms. Immunity 29, 272-282 (2008).

23. Okubo, T., Knoepfler, P.S., Eisenman, R.N. \& Hogan, B.L. Nmyc plays an essential role during lung development as a dosage-sensitive regulator of progenitor cell proliferation and differentiation. Development 132 , 1363-1374 (2005).

24. Eblaghie, M.C., Reedy, M., Oliver, T., Mishina, Y. \& Hogan, B.L. Evidence that autocrine signaling through Bmpr1a regulates the proliferation, survival and morphogenetic behavior of distal lung epithelial cells. Dev. biol. 291 67-82 (2006).

25. Lloyd, C.M. \& Saglani, S. Epithelial cytokines and pulmonary allergic inflammation. Curr. opin. immunol. 34, 52-58 (2015). 
26. Chung, Y. et al. Critical regulation of early Th17 cell differentiation by interleukin-1 signaling. Immunity 30, 576-587 (2009).

27. Bettelli, E. et al. Reciprocal developmental pathways for the generation of pathogenic effector TH17 and regulatory T cells. Nature 441, 235-238 (2006).

28. Buenrostro, J.D., Giresi, P.G., Zaba, L.C., Chang, H.Y. \& Greenleaf, W.J. Transposition of native chromatin for fast and sensitive epigenomic profiling of open chromatin, DNA-binding proteins and nucleosome position. Nat. methods 10, 1213-1218 (2013).

29. Moog-Lutz, C. et al. JAML, a novel protein with characteristics of a junctional adhesion molecule, is induced during differentiation of myeloid leukemia cells. Blood 102, 3371-3378 (2003).

30. McAlees, J.W. et al. Distinct Tlr4-expressing cell compartments control neutrophilic and eosinophilic airway inflammation. Mucosal immunol. 8, 863-873 (2015).

31. Halim, T.Y. et al. Group 2 innate lymphoid cells are critical for the initiation of adaptive T helper 2 cell-mediated allergic lung inflammation. Immunity 40 , 425-435 (2014).

32. Krishnaswamy, J.K. et al. Coincidental loss of DOCK8 function in NLRP10deficient and $\mathrm{C} 3 \mathrm{H} / \mathrm{HeJ}$ mice results in defective dendritic cell migration. Proc. Natl. Acad. Sci. USA 112, 3056-3061 (2015).

33. Mann, J., Oakley, F., Johnson, P.W. \& Mann, D.A. CD40 induces interleukin-6 gene transcription in dendritic cells: regulation by TRAF2, AP-1, NF-kappa B, AND CBF1. J. biol. chem. 277, 1712517138 (2002).

34. Perona-Wright, G. et al. A pivotal role for CD40-mediated IL-6 production by dendritic cells during IL-17 induction in vivo. J. immunol. 182, 28082815 (2009).

35. Cose, S., Brammer, C., Khanna, K.M., Masopust, D. \& Lefrancois, L. Evidence that a significant number of naive $T$ cells enter non-lymphoid organs as part of a normal migratory pathway. Eur. j. immunol. 36, 14231433 (2006).

36. Grinnan, D. et al. Enhanced allergen-induced airway inflammation in paucity of lymph node T cell (plt) mutant mice. J. allergy clin. immunol. 118, 1234-1241 (2006).

37. Nambiar, J.K., Ryan, A.A., Kong, C.U., Britton, W.J. \& Triccas, J.A. Modulation of pulmonary DC function by vaccine-encoded GM-CSF enhances protective immunity against Mycobacterium tuberculosis infection. Eur. j. immunol. 40, 153-161 (2010).

38. Averill, M.M. et al. S100A9 differentially modifies phenotypic states of neutrophils, macrophages, and dendritic cells: implications for atherosclerosis and adipose tissue inflammation. Circulation 123, 1216-1226 (2011).

39. Lambrecht, B.N. \& Hammad, H. Allergens and the airway epithelium response: gateway to allergic sensitization. J. allergy clin. immunol. 134, 499-507 (2014).

40. Dewas, C. et al. TSLP expression: analysis with a ZsGreen TSLP reporter mouse. J. immunol. 194, 1372-1380 (2015).

41. Hardman, C.S., Panova, V. \& McKenzie, A.N. IL-33 citrine reporter mice reveal the temporal and spatial expression of IL-33 during allergic lung inflammation. Eur. j. immunol. 43, 488-498 (2013).

42. Zaret, K.S. \& Carroll, J.S. Pioneer transcription factors: establishing competence for gene expression. Genes dev. 25, 2227-2241 (2011).

43. Henriques, T. et al. Stable pausing by RNA polymerase II provides an opportunity to target and integrate regulatory signals. Mol. cell 52, 517-528 (2013).

44. Willart, M.A. et al. Interleukin-1alpha controls allergic sensitization to inhaled house dust mite via the epithelial release of GM-CSF and IL-33. J. exp. med. 209, 1505-1517 (2012).

45. Santori, F.R. et al. Identification of natural RORgamma ligands that regulate the development of lymphoid cells. Cell metab. 21, 286-297 (2015).

46. $\mathrm{Hu}, \mathrm{X}$. et al. Sterol metabolism controls $\mathrm{T}(\mathrm{H}) 17$ differentiation by generating endogenous RORgamma agonists. Nat. chem. biol. 11, 141-147 (2015).

47. Draper, D.W. et al. ATP binding cassette transporter G1 deletion induces IL17-dependent dysregulation of pulmonary adaptive immunity. J. immunol. 188, 5327-5336 (2012).

48. Thery, C., Zitvogel, L. \& Amigorena, S. Exosomes: composition, biogenesis and function. Nat. rev. Immunol. 2, 569-579 (2002).

49. Villarroya-Beltri, C., Gutierrez-Vazquez, C., Sanchez-Madrid, F. \& Mittelbrunn, M. Analysis of microRNA and protein transfer by exosomes during an immune synapse. Methods mol. biol. 1024, 41-51 (2013).

50. Nakano, H., Moran, T.P., Nakano, K., Gerrish, K.E., Bortner, C.D. \& Cook, D.N. Complement receptor C5aR1/CD88 and dipeptidyl peptidase-4/ CD26 define distinct hematopoietic lineages of dendritic cells. J. immunol. 194, 3808-3819 (2015). 